\title{
IDENTITIES FOR FAMILIES OF ORTHOGONAL POLYNOMIALS AND SPECIAL FUNCTIONS
}

\author{
Wolfram KOEPF \\ Konrad-Zuse-Zentrum fr Informationstechnik Berlin, Takustr. 7, D-14195 Berlin
}

(Received June 15, 1995)

\begin{abstract}
In this article we present new results for families of orthogonal polynomials and special functions, that are determined by algorithmical approaches. In the first section, we present new results, especially for discrete families of orthogonal polynomials, obtained by an application of the celebrated Zeilberger algorithm. Next, we present algorithms for holonomic families $f(n, x)$ of special functions which possess a derivative rule. We call those families admissible. A family $f(n, x)$ is holonomic if it satisfies a holonomic recurrence equation with respect to $n$, and a holonomic differential equation with respect to $x$, i. e. linear homogeneous equations with polynomial coefficients. The rather rigid property of admissibility has many interesting consequences, that can be used to generate and verify identities for these functions by linear algebra techniques. On the other hand, many families of special functions, in particular families of orthogonal polynomials, are admissible. We moreover present a method that generates the derivative rule from the holo nomic representation of a holonomic family. As examples, we find new identities for the Jacobi polynomials and for the Whittaker functions, and for families of discrete orthogonal polynomials by the given approach. Finally, we present representations for the parameter derivatives of the Gegenbauer and the generalized Laguerre polynomials.
\end{abstract}

KEY WORDS: orthogonal polynomials, algorithmical approaches, Whittaker functions

MSC (1991): 33C45

\section{HOLONOMIC FUNCTIONS}

Let $\mathbb{K}[n, x]$ denote the polynomial ring over $\mathbb{K}$ in the variables $n$ and $x$, and $\mathbb{K}(n, x)$ the field of rational functions over $\mathbb{K}$ where $\mathbb{K}$ is one of $\mathbb{Q}, \mathbb{R}$, or $\mathbb{C}$.

Many special functions can be looked at from the following point of view: They represent functions $f(n, x)$ of one "discrete" variable $n \in \mathbb{Z}$, and one "continuous" variable $x \in I$ where $I$ represents a real interval, either finite $I=[a, b]$, infinite 
$(I=[a, \infty), I=(-\infty, a]$, or $I=\mathbb{R})$, or a subset of the complex plane $\mathbb{C}$. In the given situation we may speak of the family $\left(f_{n}\right)_{n \in \mathbf{Z}}$ of functions $f_{n}(x):=f(n, x)$.

Such a family is called a holonomic system if $f_{n}(x)$ satisfies a holonomic recurrence equation with respect to $n$, i. e. a linear homogeneous recurrence equation

$$
\sum_{k=0}^{m} p_{k}(n, x) f_{n-k}(x)=0
$$

with polynomial coefficients $p_{k} \in \mathbb{K}[n, x]$, and if it furthermore satisfies a holonomic differential equation with respect to $x$, i. e. a linear homogeneous differential equation

$$
\sum_{k=0}^{m} q_{k}(n, x) f_{n}^{(k)}(x)=0
$$

with polynomial coefficients $q_{k} \in \mathbb{K}[n, x]$.

As an example, the Legendre polynomials $f_{n}(x)=P_{n}(x)$ satisfy the holonomic recurrence equation

$$
n f_{n}(x)+(1-2 n) x f_{n-1}(x)+(n-1) f_{n-2}(x)=0
$$

and the holonomic differential equation

$$
\left(x^{2}-1\right) f_{n}^{\prime \prime}(x)+2 x f_{n}^{\prime}(x)-n(1+n) f_{n}(x)=0 .
$$

Therefore they represent a holonomic system completely determined by the two holonomic equations, and the initial values

$$
f_{0}(0)=1, \quad f_{1}(0)=0, \quad f_{0}^{\prime}(0)=0, \quad f_{1}^{\prime}(0)=1 .
$$

In recent work, Zeilberger [36] introduced holonomic systems (in a more general setting) and showed how by an elimination process the holonomic equations can be used to verify identities for holonomic systems. We will give a rigorous introduction to this approach in Section 8.

In [37]-[38], Zeilberger published an algorithm which calculates the holonomic recurrence equation for functions $\Sigma(n)$ given as infinite sums

$$
\Sigma(n):=\sum_{k \in \mathbb{Z}} F(n, k)
$$

for which $F(n, k)$ is a hypergeometric term with respect to both $n$ and $k$, i. e.

$$
\frac{F(n, k)}{F(n-1, k)}, \frac{F(n, k)}{F(n, k-1)} \in \mathbb{K}(n, k)
$$

are rational functions with respect to both $n$ and $k, n$ is assumed to be an integer, and the sum is to be taken over all integers $k \in \mathbb{Z}$. For a rigorous description of Zeilberger's algorithm, see [21]. 
Typical examples to which Zeilberger's algorithm applies are given by generalized hypergeometric functions ${ }_{p} F_{q}$

$$
{ }_{p} F_{q}\left(\begin{array}{cccc|}
a_{1} & a_{2} & \cdots & a_{p} \\
b_{1} & b_{2} & \cdots & b_{q}
\end{array} \mid x\right):=\sum_{k=0}^{\infty} A_{k} x^{k}=\sum_{k=0}^{\infty} \frac{\left(a_{1}\right)_{k} \cdot\left(a_{2}\right)_{k} \cdots\left(a_{p}\right)_{k}}{\left(b_{1}\right)_{k} \cdot\left(b_{2}\right)_{k} \cdots\left(b_{q}\right)_{k} k !} x^{k}
$$

$(a)_{k}=\frac{\Gamma(a+k)}{\Gamma(k)}$ denoting the Pochhammer symbol or shifted factorial, with upper and lower parameters $a_{k}$, and $b_{k}$ that are integer-linear in $n$.

In [15], an extension of Zeilberger's algorithm was given covering generalized hypergeometric functions with rational-linear parameters. Many examples are considered in [15].

An application of Zeilberger's algorithm to the hypergeometric representation

$$
P_{n}(x)={ }_{2} F_{1}\left(\begin{array}{cc|c}
-n, & n+1 & 1-x \\
1 & \frac{1-x}{2}
\end{array}\right)=\sum_{k=0}^{\infty} \frac{(-n)_{k}(n+1)_{k}}{k !^{2}}\left(\frac{1-x}{2}\right)^{k}
$$

of the Legendre polynomials ([1], (22.5.48)) yields (2), again.

As soon as a hypergeometric representation is known, the holonomic recurrence equation-often being a three term recurrence equation, see [1] - of any family of special functions can be obtained.

In some instances, however, those holonomic recurrence equations are not known. This is the case, for the Whittaker functions $M_{n, m}(x)$ and $W_{n, m}(x)$ (see $[1], \S 13.4$ ) with respect to their second parameter $m$, and for some families of discrete orthogonal polynomials (see [25]): the Krawtchouk polynomials $k_{n}^{(p)}(x, N)$, the Hahn type polynomials $h_{n}^{(\alpha, \beta)}(x, N)$, the discrete Chebyshev polynomials $t_{n}(x, N)$, the Meixner polynomials $m_{n}^{(\gamma, \mu)}(x)$, the discrete Laguerre polynomials $l_{n}^{(\rho, \alpha)}(x)([22]-$ [23], see [8], $\S 3.1)$ and the Charlier polynomials $c_{n}^{(\mu)}(x)$. The next theorem states these results, and we give $f_{n+1}$ in terms of $f_{n}$ and $f_{n-1}$.

Theorem 1. The Whittaker functions $M_{n, m}(x)$ satisfy the holonomic recurrence equation

$$
\begin{aligned}
M_{n, m+1}(x)= & 16 \frac{(1+2 m) m\left(4 m^{2}-1-2 n x\right)(m+1)}{x(2 m-1)(2 n+1+2 m)(2 n-1-2 m)} M_{n, m}(x) \\
& -16 \frac{(m+1) m(1+2 m)^{2}}{(2 n+1+2 m)(2 n-1-2 m)} M_{n, m-1}(x)
\end{aligned}
$$

with respect to the parameter $m$.

The Whittaker functions $W_{n, m}(x)$ satisfy the holonomic recurrence equation

$$
W_{n, m+1}(x)=\frac{4 m\left(4 m^{2}-2 n x-1\right)}{(2 m-1)(2 m+1-2 n)} W_{n, m}(x)
$$




$$
+\frac{(1+2 m)(2 m+2 n-1) x}{(2 m-1)(2 m+1-2 n)} W_{n, m-1}(x)
$$

with respect to the parameter $m$.

The Krawtchouk polynomials $k_{n}^{(p)}(x, N)$ satisfy the holonomic recurrence equations

$$
\begin{gathered}
k_{n+1}^{(p)}(x, N)=\frac{(1-n+N)(p-1) p}{1+n} k_{n-1}^{(p)}(x, N)+\frac{-n+2 n p-N p+x}{1+n} k_{n}^{(p)}(x, N) \\
k_{n}^{(p)}(x, N+1)=\frac{N-x}{(1-n+N)(p-1)} k_{n}^{(p)}(x, N-1)+\frac{n-1-2 N+p+N p+x}{(1-n+N)(p-1)} k_{n}^{(p)}(x, N) \\
k_{n}^{(p)}(x+1, N)=\frac{(p-1) x}{p(N-x)} k_{n}^{(p)}(x-1, N)+\frac{n-N p-x+2 p x}{p(x-N)} k_{n}^{(p)}(x, N)
\end{gathered}
$$

with respect to the parameters $n, N$, and $x$, respectively.

The Hahn type polynomials $h_{n}^{(\alpha, \beta)}(x, N)$ satisfy the holonomic recurrence equations

$$
\begin{aligned}
& h_{n+1}^{(\alpha, \beta)}(x, N)=\frac{(-N+n)(n+\alpha)(\beta+n)(\alpha+2 n+2+\beta)(n+\alpha+\beta+N)}{(n+1)(n+1+\alpha+\beta)(\alpha+2 n+\beta)} h_{n-1}^{(\alpha, \beta)}(x, N) \\
& +\left(2 n+4 n x \beta+4 x n \alpha+\beta+\alpha-\alpha N \beta-2 n N \beta+x \alpha^{2}-n \alpha^{2}+\beta^{2}-N \beta-2 N n+2 x \beta\right. \\
& +4 x n+3 n \beta+2 n^{2}-2 N n^{2}+4 x n^{2}+n^{2} \beta-\alpha n^{2}-N \beta^{2}+x \beta^{2}+n \beta^{2}+n \alpha-2 \alpha N n \\
& +2 \alpha x \beta+\alpha \beta+2 x \alpha-\alpha N) \frac{(1+2 n+\beta+\alpha)}{(n+1)(n+1+\alpha+\beta)(\alpha+2 n+\beta)} h_{n}^{(\alpha, \beta)}(x, N), \\
& h_{n}^{(\alpha+1, \beta)}(x, N)=\frac{(\alpha+n)(\alpha+\beta+n+N)}{(1+\alpha+\beta+n)(-\alpha-N+x)} h_{n}^{(\alpha-1, \beta)}(x, N) \\
& -\frac{\alpha+2 \alpha^{2}+2 \alpha \beta+3 \alpha n+\beta n+n^{2}+N+2 \alpha N+\beta N+2 n N-(1+\alpha+\beta+2 n) x}{(1+\alpha+\beta+n)(-\alpha-N+x)} h_{n}^{(\alpha, \beta)}(x, \Lambda \\
& h_{n}^{(\alpha, \beta+1)}(x, N)=-\frac{(\beta+n)(\alpha+\beta+n+N)}{(1+\alpha+\beta+n)(1+\beta+x)} h_{n}^{(\alpha, \beta-1)}(x, N) \\
& +\frac{1+\alpha+2 \beta+2 \alpha \beta+2 \beta^{2}+2 n+\alpha n+3 \beta n+n^{2}+\beta N+(1+\alpha+\beta+2 n) x}{(1+\alpha+\beta+n)(1+\beta+x)} h_{n}^{(\alpha, \beta}(x, N), \\
& h_{n}^{(\alpha, \beta)}(x, N+1)=\frac{(\alpha+\beta+n+N)(N-1-x)}{(N-n)(-\alpha-N+x)} h_{n}^{(\alpha, \beta)}(x, N-1)
\end{aligned}
$$




$$
\begin{gathered}
+\frac{\left(\alpha+\beta+n+\alpha n+\beta n+n^{2}+N-2 \alpha N-\beta N-2 N^{2}+(\alpha+\beta+2 N) x\right.}{(N-n)(-\alpha-N+x)} h_{n}^{(\alpha, \beta)}(x, N), \\
h_{n}^{(\alpha, \beta)}(x+1, N)=\frac{x(-\alpha-N+x)}{(N-1-x)(1+\beta+x)} h_{n}^{(\alpha, \beta)}(x-1, N) \\
-\frac{1+\beta+n+\alpha n+\beta n+n^{2}-N-\beta N+(2-\alpha+\beta-2 N) x+2 x^{2}}{(N-1-x)(1+\beta+x)} h_{n}^{(\alpha, \beta)}(x, N)
\end{gathered}
$$

with respect to the parameters $n, \alpha, \beta, N$, and $x$, respectively.

The Meixner polynomials $m_{n}^{(\gamma, \mu)}(x)$ satisfy the holonomic recurrence equations

$$
\begin{aligned}
m_{n+1}^{(\gamma, \mu)}(x)= & \frac{(1-\gamma-n) n}{\mu} m_{n-1}^{(\gamma, \mu)}(x)+\frac{\gamma \mu+n+\mu n-x+\mu x}{\mu} m_{n}^{(\gamma, \mu)}(x), \\
m_{n}^{(\gamma+1, \mu)}(x)= & \frac{-1+\gamma+n}{(\mu-1)(\gamma+x)} m_{n}^{(\gamma-1, \mu)}(x) \\
& +\frac{1-2 \gamma+\gamma \mu-n+\mu n-x+\mu x}{(\mu-1)(\gamma+x)} m_{n}^{(\gamma, \mu)}(x), \\
m_{n}^{(\gamma, \mu)}(x+1)= & !-\frac{x}{\mu(\gamma+x)} m_{n}^{(\gamma, \mu)}(x-1)+\frac{\gamma \mu-n+\mu n+x+\mu x}{\mu(\gamma+x)} m_{n}^{(\gamma, \mu)}(x)
\end{aligned}
$$

with respect to the parameters $n, \gamma$, and $x$, respectively.

The discrete Laguerre polynomials $l_{n}^{(\rho, \alpha)}(x)$ satisfy the holonomic recurrence equations

$$
\begin{aligned}
l_{n+1}^{(\rho, \alpha)}(x) & =-\frac{\alpha+n}{1+n} l_{n-1}^{(\rho, \alpha)}(x)+\frac{1+n+\alpha \rho+n \rho-x+\rho x}{1+n} l_{n}^{(\rho, \alpha)}(x), \\
l_{n}^{(\rho, \alpha+1)}(x) & =\frac{\alpha+n}{(\rho-1)(\alpha+x)} l_{n}^{(\rho, \alpha-1)}(x)+\frac{-2 \alpha-n+\alpha \rho+n \rho-x+\rho x}{(\rho-1)(\alpha+x)} l_{n}^{(\rho, \alpha)}(x) \\
l_{n}^{(\rho, \alpha)}(x+1) & =\frac{1-x}{\rho(\alpha+x)} l_{n}^{(\rho, \alpha)}(x-1)+\frac{-1-n+\alpha \rho+n \rho+x+\rho x}{\rho(\alpha+x)} l_{n}^{(\rho, \alpha)}(x)
\end{aligned}
$$

with respect to the parameters $n, \alpha$, and $x$, respectively.

The Charlier polynomials $c_{n}^{(\mu)}(x)$ satisfy the holonomic recurrence equations

$$
\begin{aligned}
c_{n+1}^{(\mu)}(x) & =-\frac{n}{\mu} c_{n-1}^{(\mu)}(x)+\frac{\mu+n-x}{\mu} c_{n}^{(\mu)}(x), \\
c_{n}^{(\mu)}(x+1) & =-\frac{x}{\mu} c_{n}^{(\mu)}(x-1)+\frac{\mu-n+x}{\mu} c_{n}^{(\mu)}(x)
\end{aligned}
$$

with respect to the parameters $n$, and $x$, respectively. 
Proof. Zeilberger's algorithm generates the results when applied to the hypergeometric representations

$$
M_{n, m}(x)=e^{-x / 2} x_{1}^{1 / 2+m} F_{1}\left(\begin{array}{c}
1 / 2+m-n \\
1+2 m
\end{array}\right)
$$

$([1],(13.1 .32))$

$$
k_{n}^{(p)}(x, N)=(-1)^{n} p^{n}\left(\begin{array}{c}
N \\
n
\end{array}\right){ }_{2} F_{1}\left(\begin{array}{cc|c}
-n, & -x & 1 \\
-N & p
\end{array}\right)
$$

$([25],(2.7 .11 \mathrm{a}))$

$$
\begin{aligned}
& h_{n}^{(\alpha, \beta)}(x, N)
\end{aligned}
$$

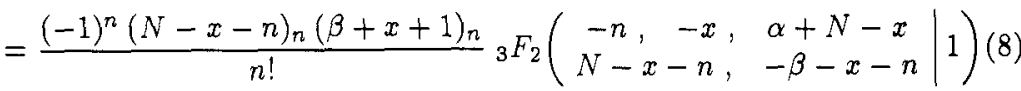

$$
\begin{aligned}
& =\frac{(-1)^{n}(N-n)_{n}(\beta+1)_{n}}{n !}{ }_{3} F_{2}\left(\begin{array}{cc|c}
-n, & -x, & \alpha+\beta+n+1 \\
\beta+1, & 1-N
\end{array} \mid 1\right) \text {, }
\end{aligned}
$$

(compare [25], (2.7.19)),

$$
m_{n}^{(\gamma, \mu)}(x)=(\gamma)_{n} \cdot{ }_{2} F_{1}\left(\begin{array}{cc|c}
-n, & -x & 1-\frac{1}{\mu} \\
\gamma & & 1-
\end{array}\right.
$$

(see [9], $10.24(9),[25],(2.7 .13))$,

$$
l_{n}^{(\rho, \alpha)}(x)=\frac{\rho^{n}}{n !} m_{n}^{(1+\alpha, \rho)}(x-1)=\frac{\rho^{n}}{n !}(1+\alpha)_{n} \cdot{ }_{2} F_{1}\left(\begin{array}{cc}
-n, & -x+1 \\
\alpha+1 & 1-\frac{1}{\rho}
\end{array}\right),
$$

(see $[35],(2.18)-(2.20))$, and

$$
c_{n}^{(\mu)}(x)={ }_{2} F_{0}(-n, \quad-x \mid-1 / \mu)=\frac{(-1)^{n}}{\mu^{n}}(x-n+1)_{n 1} F_{1}\left(\begin{array}{c}
-n \\
-x-n+1
\end{array} \mid \mu\right),
$$

([25], (2.7.9)).

For the Whittaker functions $W_{n, m}(x)$ the recurrence equation is obtained by a different method. Since by

$$
W_{n, m}(x)=\frac{\Gamma(-2 m)}{\Gamma(1 / 2-m-n)} M_{n, m}(x)+\frac{\Gamma(2 m)}{\Gamma(1 / 2+m-n)} M_{n,-m}(x)
$$


([1], (13.1.32)) $W_{n, m}(x)$ is represented as sum of products, the recurrence equation can be obtained from (5), and the recurrence equation of the $\Gamma$ function (see [29], $[36],[20],[28])$.

We note that similarly, one can obtain holonomic recurrence equations for the Hahn type polynomials $\tilde{h}_{n}^{(\mu, \nu)}(x, N)$ (see [25], $\S 2.4$ ), and $p_{n}(x, \beta, \gamma, \delta)$ (see [9], $\S 10.23)$.

Note further that some of the above recurrence equations have appeared in the literature. Relation (6), for $\alpha=\beta=0$ is a recurrence equation for the discrete Chebyshev polynomials $t_{n}(x, N)=h_{n}^{(0,0)}(x, N)$ (compare [9], $10.23(10)$, and [25], $\S 2.4)$, and can be found in ([9], $\S 10.23,(6))$.

However, these relations nowhere appeared systematically. The important issue of our presentation is its algorithmic content: All given representations can be calculated by a computer algebra system, by implementations in MATHEMATICA ([26] and [17]), Reduce [16], and Maple [15]. Note that in our Mathematica implementation [17] all the partial algorithms mentioned are applied completely automatically.

\section{APPLICATION TO FEYNMAN DIAGRAMS}

Zeilberger's algorithm can be applied to find hypergeometric identities (see [17]), and as shown in the last section, to find three-term recurrence equations for families of orthogonal polynomials.

Here, we give another application: In ([10], see Equation (31)) the hypergeometric function

$$
\begin{aligned}
V(\alpha, \beta, \gamma):= & (-1)^{\alpha+\beta+\gamma} \frac{\Gamma(\alpha+\beta+\gamma-d / 2) \Gamma(d / 2-\gamma) \Gamma(\alpha+\gamma-d / 2) \Gamma(\beta+\gamma-d / 2)}{\Gamma(\alpha) \Gamma(\beta) \Gamma(d / 2) \Gamma(\alpha+\beta+2 \gamma-d) M^{\alpha+\beta+\gamma-d}} \\
& \times{ }_{2} F_{1}\left(\begin{array}{c}
\alpha+\beta+\gamma-d, \alpha+\gamma-d / 2 \\
\alpha+\beta+2 \gamma-d
\end{array} \mid z\right)
\end{aligned}
$$

plays an important role for the calculation of certain Feynman diagrams. It is of both theoretical and practical interest that $V(\alpha, \beta, \gamma)$ satisfies three-term recurrence equations with respect to $\alpha, \beta$, and $\gamma$. Zeilberger's algorithm yields

Theorem 2. For the function $V(\alpha, \beta, \gamma)$, given by (13), the recurrence equations

$0=(2 \alpha-d+2 \gamma)(2 \alpha+2 \beta-d+2 \gamma)(2+2 \alpha+2 \beta-d+2 \gamma) V(\alpha, \beta, \gamma)$

$$
\begin{aligned}
& -2 \alpha(2+2 \alpha+2 \beta-d+2 \gamma) M(-2 \alpha-2 \beta+2 d-4 \gamma+2 z+4 \alpha z+2 \beta z-3 d z+4 \gamma z) \\
& \times V(1+\alpha, \beta, \gamma)+8 \alpha(1+\alpha)(1+\alpha+\beta-d+\gamma) M^{2}(-1+z) \\
& \times z V(2+\alpha, \beta, \gamma)
\end{aligned}
$$




$$
\begin{gathered}
0=(2 \beta-d+2 \gamma)(2 \alpha+2 \beta-d+2 \gamma)(2+2 \alpha+2 \beta-d+2 \gamma) \\
\quad \times V(\alpha, \beta, \gamma)-2 \beta(2+2 \alpha+2 \beta-d+2 \gamma) \\
\times M(-2 \alpha-2 \beta+2 d-4 \gamma-2 z-2 \beta z+d z) V(\alpha, 1+\beta, \gamma) \\
+8 \beta(1+\beta)(1+\alpha+\beta-d+\gamma) M^{2} z V(\alpha, 2+\beta, \gamma) \\
\text { and } \quad \\
0 \quad(2 \alpha-d+2 \gamma)(2+\alpha+\beta-d+2 \gamma)(2 \beta-d+2 \gamma)(2 \alpha+2 \beta-d+2 \gamma) \\
\times(2+2 \alpha+2 \beta-d+2 \gamma) V(\alpha, \beta, \gamma)+2(2-d+2 \gamma)(1+\alpha+\beta-d+2 \gamma) \\
\times(2+2 \alpha+2 \beta-d+2 \gamma) M\left(-4 \alpha-2 \alpha^{2}-4 \beta-4 \alpha \beta-2 \beta^{2}+4 d+4 \alpha d+4 \beta d\right. \\
\quad-2 d^{2}-8 \gamma-8 \alpha \gamma-8 \beta \gamma+8 d \gamma-8 \gamma^{2}+2 \alpha z+2 \beta z+2 \alpha \beta z+2 \beta^{2} z-2 d z-\alpha d z-3 \beta d z \\
\left.+d^{2} z+4 \gamma z+4 \alpha \gamma z+4 \beta \gamma z-4 d \gamma z+4 \gamma^{2} z\right) V(\alpha, \beta, 1+\gamma)+4(1+\gamma)(1+\alpha+\beta-d+ \\
\times(2-d+2 \gamma)(4-d+2 \gamma)(\alpha+\beta-d+2 \gamma) M^{2} z^{2} V(\alpha, \beta, 2+\gamma)
\end{gathered}
$$

are valid.

\section{ADMISSIBLE FAMILIES}

In this section, we present yet another approach for holonomic systems of a special type, which gives us the opportunity to generate identities other than holonomic recurrence equations by linear algebra techniques.

We assume that a holonomic system satisfies a derivative rule of the form

$$
f_{n}^{\prime}(x)=\frac{\partial}{\partial x} f_{n}(x)=\sum_{k=0}^{m-1} r_{k}(n, x) f_{n-k}(x) \text { or } f_{n}^{\prime}(x)=\sum_{k=0}^{m-1} r_{k}(n, x) f_{n+k}(x)
$$

where the derivative with respect to $x$ is represented by a finite number of lower or higher indexed functions of the family, and where $r_{k} \in \mathbb{K}(n, x)$ are rational functions in $n$ and $x$. We call the two different types of derivative rules backward and forward derivative rule, respectively.

Note that by an iterative application of (1), the order $m$ of the derivative rule (13) can be made less than or equal to the order $m$ of the recurrence equation (1). 
This is our general assumption.

From an algebraic point of view these properties read as follows: If the coefficients of the occurring polynomials and rational functions are elements of $\mathbb{K}$, then

1. the derivative rule states that $f_{n}^{\prime}$ is an element of the linear space over $\mathbb{K}(n, x)$ which is generated by $\left\{f_{n}, f_{n-1}, \ldots, f_{n-(m-1)}\right\}$ or $\left\{f_{n}, f_{n+1}, \ldots, f_{n+m-1}\right\}$, respectively;

2. the holonomic differential equation states that the $m+1$ functions $f_{n}^{(k)}(k=$ $0, \ldots, m)$ are linearly dependent over $\mathbb{K}(n, x)$; moreover, by an induction argument, any $m+1$ functions $f_{n}^{(k)}\left(k \in \mathbb{N}_{0}\right)$ are linearly dependent over $\mathbb{K}(n, x)$;

3. the holonomic recurrence equation states that the $m+1$ functions $f_{n-k}(k=$ $0, \ldots, m)$ are linearly dependent over $\mathbb{K}(n, x)$; moreover, by an induction argument, any $m+1$ functions $f_{n}(n \in \mathbb{Z})$, are linearly dependent over $\mathbb{K}(n, x)$.

Our main notion is the

Definition 1. (Admissible family of special functions) We call a family $f_{n}$ of functions admissible if they satisfy a recurrence equation of type (1) and a derivative rule of type (13). We call the order of the recurrence equation the order of the admissible family $f_{n}$

The recurrence equation (1) together with $m$ initial functions $f_{n_{0}}, f_{n_{0}+1}, \ldots$, $f_{n_{0}+m-1}$ determine the functions $f_{n}(n \in \mathbb{Z})$ uniquely.

Therefore an admissible family of special functions (with given initial functions) is overdetermined by its two defining properties, i. e. the recurrence equation and the derivative rule must be compatible. This fact, however, gives our notion a considerable strength:

Theorem 3. For any admissible family $f_{n}$ of order $m$ the linear space $V_{f_{n}}$ over $\mathbb{K}(n, x)$ of functions generated by the set of shifted derivatives $\left\{f_{n \pm k}^{(j)} \mid j, k \in \mathbb{N}_{0}\right\}$ is at most m-dimensional. On the other hand, if the family $\left\{f_{n \pm k}^{(j)} \mid j, k \in \mathbb{N}_{0}\right\}$ spans an $m$-dimensional linear space over $\mathbb{K}(n, x)$, then $f_{n}$ forms an admissible family of order $m$.

Proof. By the recurrence equation and an induction argument it follows that the linear space $V$ spanned by $\left\{f_{n \pm k} \mid k \in \mathbb{N}_{0}\right\}$ is at most $m$-dimensional. Using the derivative rule, by a further induction it follows that the derivative of any order $f_{n}^{(k)}\left(k \in \mathbb{N}_{0}\right)$ is an element of $V$. Therefore $V_{f_{n}}=V$.

If on the other hand for a family $f_{n}$ the set of derivatives $\left\{f_{n \pm k}^{(j)} \mid j, k \in \mathbb{N}_{0}\right\}$ is $m$-dimensional, then the existence of a recurrence equation and a derivative rule of order $m$ are obvious.

Note that it can happen that $V$ has dimension less than $m$. Assume $f_{n}(x)=e^{x}$ is given as admissible family by $f_{n}(x)-f_{n-2}(x)=0$ and $f_{n}^{\prime}(x)=f_{n}(x)$. Then the family of shifted derivatives $\left\{f_{n \pm k}^{(j)} \mid j, k \in \mathbb{N}_{0}\right\}$ consists just of $f_{n}(x)$, and is therefore one-dimensional rather than two-dimensional. This is due to the fact that the 
representing recurrence equation is not of lowest possible order. To guarantee that $V$ is $m$-dimensional it is therefore necessary to assume that $f_{n}, f_{n-1}, \ldots, f_{n-m+1}$ are linearly independent over $\mathbb{K}(n, x)$.

The following consequence of Theorem 3 is the main reason for the importance of admissible families: Any $m+1$ distinguished elements of $V_{f_{n}}$ are linearly dependent, i. e. any arbitrary element of $V_{f_{n}}$ can be represented by a linear combination (with respect to $\mathbb{K}(n, x))$ of any $m$ of the others. This is the algebraic background for the fact that so many identities between the members and their derivatives of an admissible family exist.

In particular we have

Corollary 1. Any admissible family $f_{n}$ of order $m$ satisfies a holonomic differential equation of order $m$, and therefore constitutes a holonomic system.

With regard to Zeilberger's approach, Corollary 1 can be interpreted as follows: Any admissible family $f_{n}(x)$ forms a holonomic system with respect to the two variables $n$ and $x$, whose defining recurrence equation, and the differential equation corresponding to Corollary 1 together with the initial conditions

$$
f_{0}^{(k)}(0), \quad \text { and } \quad f_{k}(0) \quad(k=0, \ldots, m-1)
$$

yield the canonical holonomic representation of $f_{n}(x)$ (see [36], Lemma 4.1).

On the other hand, not all holonomic systems $f_{n}(x)$ form admissible families so that our notion is stronger: Let $f_{n}(x):=\mathrm{Ai}(x)$ be the Airy function (see [1], $\S 10.4)$ for all $n \in \mathbb{Z}$, then obviously $f_{n}(x)$ is the holonomic system generated by the equations

$$
f_{n}^{\prime \prime}(x)=x f_{n}(x), \quad f_{n+1}(x)=f_{n}(x)
$$

and some initial values, that does not form an admissible family since the derivative $f_{n}^{\prime}=\mathrm{Ai}^{\prime}$ is linearly independent of $f_{n}$ over $\mathbb{K}(n, x)$, and thus no derivative rule of the form (13) exists.

Looking in mathematical dictionaries like [1], one realizes that the class of admissible families is large. Besides the exponential, sine and cosine functions, it contains the Airy functions $\mathrm{Ai}(x), \mathrm{Bi}(x)$ (see [1], $\S 10.4$ ), the exponential integrals $E_{n}(x)$ (see [1], (5.1)), the iterated integrals of the (complementary) error function $\operatorname{erfc}_{n}(x)$ (see [1], $(7.2)$ ), the Bessel functions $J_{n}(x), Y_{n}(x), I_{n}(x)$, and $K_{n}(x)$ (see [1], Ch. 9-11), the Hankel functions $H_{n}^{(1)}(x)$ and $H_{n}^{(2)}(x)$ (see [1], Ch. 9), the Kummer functions $M(a, b, x)={ }_{1} F_{1}\left(\begin{array}{c}a \\ b\end{array} \mid x\right)$ and $U(a, b, x)$ (see [1], Ch. 13), the Whittaker functions $M_{n, m}(x)$ and $W_{n, m}(x)$ (see [1], $\left.\S 13.4\right)$, the associated Legendre functions $P_{a}^{b}(x)$ and $Q_{a}^{b}(x)$ (see [1], $\S 8$ ), the Struve functions $\mathbf{H}_{n}(x)$ and $\mathrm{L}_{n}(x)$ (see [1], Chapter 5), all kinds of orthogonal polynomials: the Jacobi polynomials $P_{n}^{(\alpha, \beta)}(x)$, the Gegenbauer polynomials $C_{n}^{(\alpha)}(x)$, the Chebyshev polynomials of the first kind $T_{n}(x)$ and of the second kind $U_{n}(x)$, the Legendre polynomials $P_{n}(x)$, the Laguerre polynomials $L_{n}^{(\alpha)}(x)$, and the Hermite polynomials $H_{n}(x)$ (see [30], [34], and [1], 
$\S 22$ ), and many more special functions. The defining recurrence equations and derivative rules of the above functions are listed in [14].

To present an example of an admissible family that cannot be found in mathematical dictionaries (see, however, [1] (13.6)), we consider the functions

$$
k_{n}(x):=\frac{2}{\pi} \int_{0}^{\pi / 2} \cos (x \tan \theta-n \theta) d \theta
$$

that Bateman introduced in [4], see also [19]. He verified that ([4], formula (2.7))

$$
F_{n}(x):=(-1)^{n} k_{2 n}(x)=(-1)^{n} e^{-x}\left(L_{n}(2 x)-L_{n-1}(2 x)\right) .
$$

We call $F_{n}$ the Bateman functions that turn out to generate an admissible family of order two.

Bateman obtained the property ([4], formula (4.1))

$$
(n-1)\left(F_{n}(x)-F_{n-1}(x)\right)+(n+1)\left(F_{n}(x)-F_{n+1}(x)\right)=2 x F_{n}(x)
$$

leading to

$$
n F_{n}(x)-2(n-1-x) F_{n-1}(x)+(n-2) F_{n-2}(x)=0
$$

which is a holonomic recurrence equation of order two that determines the Bateman functions uniquely using the two initial functions

$$
F_{0}(x)=e^{-x} \quad \text { and } \quad F_{1}(x)=-2 x e^{-x}
$$

which follow from (15).

Bateman obtained further a difference differential equation ([4], formula (4.2))

$$
(n+1) F_{n+1}(x)-(n-1) F_{n-1}(x)=2 x F_{n}^{\prime}(x),
$$

which can be brought into the form

$$
F_{n}^{\prime}(x)=\frac{1}{x}\left((n-x) F_{n}(x)-(n-1) F_{n-1}(x)\right)
$$

using (16). This is a derivative rule of the form (13). Therefore the functions $F_{n}(x)$ form an admissible family of order two.

\section{PROPERTIES OF ADMISSIBLE FAMILIES}

It is well-known (see [29], [36], [20], [28]) that if the functions $f_{n}, g_{n}$ satisfy holonomic recurrence equations of order $m$ and $l$, respectively, then the sum and product satisfy holonomic recurrence equations of order $\leq m+l$, and $\leq m l$, respectively. We call the two functions $f_{n}$ and $g_{n}$ sum-independent (product-independent) if the resulting recurrence equation of lowest order has maximal order, i. e. order $m+l$ in the sum case, and order $m l$ in the product case. 
With respect to admissible families, we get then

Theorem 4. Let $f_{n}$ form an admissible family of order $m$. Then

(a) (Shift) $f_{n \pm k}(k \in \mathbb{N})$ forms an admissible family of order $m$;

(b) (Derivative) $f_{n}^{\prime}$ forms an admissible family of order $\leq m$;

(c) (Composition) $f_{n}$ or forms an admissible family of order $\leq m$, if $r \in \mathbb{K}(x)$.

If furthermore $g_{n}$ forms an admissible family of order $l$, then moreover

(d) (Sum) $f_{n}+g_{n}$ forms an admissible family of order $m+l$ if $f_{n}$ and $g_{n}$ are sum-independent;

(e) (Product) $f_{n} g_{n}$ forms an admissible family of order $m l$ if $f_{n}$ and $g_{n}$ are product-independent.

Proof.

(a): This is an obvious consequence of Theorem 3 .

(b): Let $g_{n}:=f_{n}^{\prime}$. We start with the recurrence equation for $f_{n}$ and take derivative to get

$$
\sum_{k=0}^{m} p_{k}^{\prime}(n, x) f_{n-k}(x)+\sum_{k=0}^{m} p_{k}(n, x) f_{n-k}^{\prime}(x)=0 .
$$

From Theorem 3, we know that each of the functions $f_{n-j}(j=0, \ldots, m)$ can be represented as a linear combination of the functions $f_{n-k}^{\prime}(k=0, \ldots, m-1)$ over $\mathbb{K}(n, x)$, which generates a holonomic recurrence equation for $g_{n}$. Similarly a derivative rule for $g_{n}$ is obtained.

(c): For the composition $h_{n}:=f_{n} \circ r$ with a rational function $r$, the recurrence equation is obtained by substitution, and the derivative rule is a result of the chain rule.

(d): By a simple algebraic argument, we see that $f_{n-k}+g_{n-k}(k \in \mathbb{Z})$ span the linear space $V:=V_{f_{n}+g_{n}}=V_{f_{n}}+V_{g_{n}}$ of dimension $\leq m+l$ over $\mathbb{K}(n, x)$. Therefore $f_{n}+g_{n}$ satisfies a holonomic recurrence equation of order $\leq m+l$. By our assumption, the dimension of $V$ is maximal, i. e. $m+l$. If we add the derivative rules for $f_{n}$ and $g_{n}$, we see that $f_{n}^{\prime}+g_{n}^{\prime} \in V$, and thus can be represented in the desired way.

(e): By a similar algebraic argument (see [29], Theorem 2.3) we see that $f_{n-k}$. $g_{n-k}(k \in \mathbb{Z})$ span a linear space $V$ of dimension $\leq m l$ over $\mathbb{K}(n, x)$, hence $f_{n} g_{n}$ satisfies a recurrence equation of order $\leq m l$. By our assumption, the dimension of $V$ is maximal, i. e. $m l$. By the product rule, and the derivative rules for $f_{n}$ and $g_{n}$ we see that the derivative of $f_{n} g_{n}$ is represented by products of the form $f_{n-k} g_{n-j}(k, j \in \mathbb{Z}$ ), and as those span the linear space $V$ (see [20], Theorem 3 (d)), we are done.

As an application we again may state that the Bateman functions form an admissible family: Using the theorem, this follows immediately from representation (15), and the admissibility of the Laguerie polynomials. 
As an example of a sum-dependent case, we consider $f_{n}(x)=e^{x}$, given by the relations $f_{n}(x)-f_{n-1}(x)=0$, and $f_{n}^{\prime}(x)=f_{n}(x)$, and $g_{n}(x)=e^{-x}$, given by the relations $g_{n}(x)-g_{n-1}(x)=0$, and $g_{n}^{\prime}(x)=-g_{n}(x)$. Both $f_{n}$ and $g_{n}$ form admissible families of order 1 , and since they both satisfy the same recurrence equation, their sum satisfies this recurrence equation of order one, too, so that they are not sum-independent. The derivative of $h_{n}=f_{n}+g_{n}$ is given by

$$
h_{n}^{\prime}=f_{n}^{\prime}+g_{n}^{\prime}=f_{n}-g_{n}
$$

and the question is whether or not this is expressible in terms of $h_{n}$ and $h_{n-1}$. These are given by

$$
h_{n}=f_{n}+g_{n} \quad \text { and } \quad h_{n-1}=f_{n-1}+g_{n-1}=f_{n}+g_{n}
$$

and therefore $h_{n-k}=f_{n}+g_{n}=h_{n}$ for all $k \in \mathbb{Z}$. Obviously $h_{n}^{\prime}=f_{n}-g_{n}$ cannot be expressed in terms of $f_{n}+g_{n}$ alone, so that $h_{n}$ does not satisfy a derivative rule of any order.

Similarly one shows that the admissible families $f_{n}(x)=\sin ^{(n)}(x)$, given by $f_{n}^{\prime}=-f_{n-1}$, and $f_{n}=-f_{n-2}$, and $g_{n}(x)=f_{n}(x)$ are not product-independent and for $h_{n}=f_{n}^{2}$ no derivative rule is valid.

Next we study algorithmic versions of the theorem. The following algorithm generates a representation of the members $f_{n \pm k}(k=0, \ldots, m-1)$ of an admissible family in terms of the derivatives $f_{n \pm j}^{\prime}(j=0, \ldots, m-1)$. By Theorem 3 we know that such a representation exists. Without loss of generality, we assume that the admissible family is given by a backward derivative rule. In the case of a forward derivative rule, a similar algorithm is valid.

Algorithm 1. Let $f_{n}$ be an admissible family of order $m$, given by a backward derivative rule

$$
f_{n}^{\prime}(x)=\sum_{k=0}^{m-1} r_{k}(n, x) f_{n-k}(x)
$$

Then the following algorithm generates a list of backward rules $(k=0, \ldots, m-1)$

$$
f_{n-k}(x)=\sum_{j=0}^{m-1} R_{j}^{k}(n, x) f_{n-j}^{\prime}(x)
$$

$\left(R_{j}^{k} \in \mathbb{K}(n, x)\right)$ for $f_{n-k}(k=0, \ldots, m-1)$ in terms of the derivatives $f_{n-j}^{\prime}(j=$ $0, \ldots, m-1)$ :

1. Shift the derivative rule $m-1$ times to obtain the set of $m$ equations

$$
f_{n-j}^{\prime}(x)=\sum_{k=0}^{m-1} r_{k}(n-j, x) f_{n-j-k}(x) \quad(j=0, \ldots, m-1)
$$

2. Utilize the recurrence equation to express all expressions on the right hand sides 
of these equations in terms of $f_{n-k}(k=0, \ldots, m-1)$ leading to

$$
f_{n-j}^{\prime}(x)=\sum_{k=0}^{m-1} r_{k}^{j}(n, x) f_{n-k}(x) \quad\left(j=0, \ldots, m-1, r_{k}^{j} \in \mathbb{K}(n, x)\right) .
$$

3. Solve this linear equations system for the variables $f_{n-k}(k=0, \ldots, m-1)$ to obtain the representations (19) searched for.

The proof of the algorithm is obvious. It is also clear how the method can be adapted to obtain forward rules in terms of the derivatives. As an example, the algorithm generates the representations

$$
F_{n}(x)=\frac{1-n+x}{2 n-1-x} F_{n}^{\prime}(x)+\frac{n-1}{2 n-1-x} F_{n-1}^{\prime}(x),
$$

and

$$
F_{n}(x)=\frac{1+n-x}{1+2 n-x} F_{n}^{\prime}(x)-\frac{1+n}{1+2 n-x} F_{n+1}^{\prime}(x)
$$

for the Bateman functions in terms of their derivatives.

We note that by means of Algorithm 1 and the results of [20] (see also [36], p. 342, and [28]), we are able to state algorithmic versions of the statements of Theorem 4.

Algorithm 2. The following algorithms lead to the derivative rules and recurrence equations of the admissible families presented in Theorem 4:

(a) (Shift) Direct use of derivative rule and recurrence equation lead to the derivative rule and the recurrence equation for $f_{n \pm 1}$; a recursive application gives the results for $f_{n \pm k}(k \in \mathbb{N})$.

(b) (Derivative) By Algorithm 1 we may replace all occurrences of $f_{n-k}$ ( $k=$ $0, \ldots, m)$ in $(18)$ by derivatives, resulting in the recurrence equation for $f_{n}^{\prime} ;$ similarly the derivative rule is obtained.

(c) (Composition) If $r$ is a rational function, then an application of the chain rule leads to the derivative rule and the recurrence equation of $f_{n} \circ r$.

(d) (Sum) Applying a discrete version of Theorem 3 (c) in [20] to $f_{n}+g_{n}$ (see also [36], p. 342, and [28], MAPLE function rectrec) results in the recurrence equation, and a similar approach gives the derivative rule.

(e) (Product) Applying a discrete version of Theorem 3 (d) in [20] to $f_{n} g_{n}$ (see also [36], p. 342, and [28], MAPLE function rec*rec) yields the recurrence equation, and a similar approach gives the derivative rule.

A Mathematica implementation [17] of the given algorithms generate for the derivative $F_{n}^{\prime}(x)$ of the Bateman function $F_{n}(x)$ the derivative rule

$$
F_{n}^{\prime \prime}(x)=\frac{2 n-x}{x-2 n x+x^{2}}\left((n-1) F_{n-1}^{\prime}(x)+(1-n+x) F_{n}^{\prime}(x)\right),
$$


and the recurrence equation

$$
F_{n+1}^{\prime}(x)=\frac{\left((n-1)(x-2 n-1) F_{n-1}^{\prime}(x)+2\left(1-2 n^{2}+3 n x-x^{2}\right) F_{n}^{\prime}(x)\right)}{\frac{1}{(1+n)(1-2 n+x)}},
$$

and for the product $A_{n}(x):=F_{n}^{2}(x)$ the derivative rule

$$
\begin{aligned}
A_{n}^{\prime}(x)= & \frac{(n-1)(n-2)^{2}}{2 n x(1-n+x)} A_{n-2}(x)-\frac{2(n-1)(1-n+x)}{n x} A_{n-1}(x) \\
& -\frac{\left(-5 n+5 n^{2}+4 x-8 n x+4 x^{2}\right)}{2(1-n+x) x} A_{n}(x),
\end{aligned}
$$

and the recurrence equation

$$
\begin{aligned}
A_{n+1}(x)= & \frac{1}{(1+n)^{2}}\left(\frac{(n-2)^{2}(n-1)(x-n)}{n(1-n+x)} A_{n-2}\right. \\
& +\frac{(n-1)\left(3 n-3 n^{2}-4 x+8 n x-4 x^{2}\right)}{n} A_{n-1} \\
& \left.+\frac{(x-n)\left(-3 n+3 n^{2}+4 x-8 n x+4 x^{2}\right)}{1-n+x} A_{n}\right)
\end{aligned}
$$

are derived.

\section{HYPERGEOMETRIC FUNCTIONS AS ADMISSIBLE FAMILIES}

An important example of an admissible family is given by the generalized hypergeometric function ${ }_{p} F_{q}$. The generalized hypergeometric function satisfies a derivative rule of order two with respect to any of its numerator parameters $a_{k}(k=1, \ldots, p)$, and denominator parameters $b_{k}(k=1, \ldots, q)$.

We choose one of the numerator parameters $n:=a_{k}(k=1, \ldots, p)$ of ${ }_{p} F_{q}$ as parameter $n$, and use the abbreviations

$$
f_{n}(x)={ }_{p} F_{q}\left(\begin{array}{c}
a_{1}, a_{2}, \cdots, n, \cdots, a_{p} \\
b_{1}, b_{2}, \cdots, b_{q}
\end{array} \mid x\right)=\sum_{k=0}^{\infty} A_{k}(n) x^{k} .
$$

From the relation

it follows that

$$
\frac{(n+1)_{k}}{(n)_{k}}=\frac{n+k}{n}
$$

$$
n A_{k}(n+1)=(n+k) A_{k}(n) .
$$

Using the differential operator $\theta f(x)=x f^{\prime}(x)$, we get by summation

$$
n f_{n+1}(x)=n \sum_{k=0}^{\infty} A_{k}(n+1) x^{k}=(n+k) \sum_{k=0}^{\infty} A_{k}(n) x^{k}
$$




$$
=n f_{n}(x)+\sum_{k=0}^{\infty} k A_{k}(n) x^{k}=n f_{n}(x)+\theta f_{n}(x),
$$

and therefore we are led to the derivative rule

$$
\theta f_{n}(x)=n\left(f_{n+1}(x)-f_{n}(x)\right), \quad \text { or } \quad f_{n}^{\prime}(x)=\frac{n}{x}\left(f_{n+1}(x)-f_{n}(x)\right) .
$$

Hence we have established that for any of the numerator parameters $n:=a_{k}$ ( $k=$ $1, \ldots, p$ ) of $F_{q}$ such a simple (forward) derivative rule is valid.

We note that by similar means for each of the denominator parameters $n:=$ $b_{k}(k=1, \ldots, q)$ of $F_{q}$ the simple (backward) derivative rule

$$
\theta f_{n}(x)=(n-1)\left(f_{n-1}(x)-f_{n}(x)\right), \text { or } f_{n}^{\prime}(x)=\frac{n-1}{x}\left(f_{n-1}(x)-f_{n}(x)\right)
$$

is derived.

Next, we note that $f_{n}$ satisfies the well-known hypergeometric differential equation

$$
\theta\left(\theta+b_{1}-1\right) \cdots\left(\theta+b_{q}-1\right) f_{n}(x)=x\left(\theta+a_{1}\right)\left(\theta+a_{2}\right) \cdots\left(\theta+a_{p}\right) f_{n}(x) .
$$

Replacing all occurrences of $\theta$ in (22) recursively by the derivative rule (20) or (21), a recurrence equation for $f_{n}$ is obtained having the order of the differential equation $(22)$, i. e. $\max \{p, q+1\}$.

We summarize the above results in the following Theorem (see [27]).

Theorem 5. The generalized hypergeometric function ${ }_{p} F_{q}\left(\begin{array}{cccc|c}a_{1} & a_{2} & \cdots & a_{p} \\ b_{1} & b_{2} & \cdots & b_{q} & x\end{array}\right)$ satisfies the derivative rules

$$
\theta f_{n}(x)=n\left(f_{n+1}(x)-f_{n}(x)\right)
$$

for any of its numerator parameters $n:=a_{k}(k=1, \ldots, p)$, and

$$
\theta f_{n}(x)=(n-1)\left(f_{n-1}(x)-f_{n}(x)\right)
$$

for any of its denominator parameters $n:=b_{k}(k=1, \ldots, q)$, and recursive substitution of all occurrences of $\theta$ in the hypergeometric differential equation

$$
\theta\left(\theta+b_{1}-1\right) \cdots\left(\theta+b_{q}-1\right) f_{n}(x)=x\left(\theta+a_{1}\right)\left(\theta+a_{2}\right) \cdots\left(\theta+a_{p}\right) f_{n}(x)
$$

generates a holonomic recurrence equation of order $\max \{p, q+1\}$ with respect to the parameter chosen. After multiplication by the common denominator, this recurrence equation has coefficients in $\mathbb{K}[n, x]$, that are linear with respect to $x$. In particular, ${ }_{p} F_{q}$ forms an admissible family of order $\max \{p, q+1\}$ with respect to any of its parameters $a_{k}, b_{k}$.

We note that if some of the parameters of ${ }_{p} F_{q}$ are specified, there may exist a lower order differential equation, and thus the order of the admissible family may 
be lower than the theorem states. We note further that this theorem is the main reason for the fact that so many special functions form admissible families: Most of them can be represented in terms of generalized hypergeometric functions.

Note that Zeilberger's algorithm determines the recurrence equation for ${ }_{p} F_{q}$ even in the case that the upper and lower parameters are integer-linear in $n$, and a generalization of Zeilberger's algorithm [15] is successful if the parameters are rational-linear in $n$, but this approach does not lead to a derivative rule.

On the other hand, one can formulate an algorithm similar (but more complicated) to the one described in this section to generate a derivative rule for such ${ }_{p} F_{q}$ which is covered by our Mathematica implementation [17]. However, in Section 8 we will present a more general approach for the same purpose, based on Gröbner basis techniques.

\section{ALGORITHMIC GENERATION OF IDENTITIES}

Since in an admissible family, the linear space spanned by the set of shifted derivatives

$V_{f_{n}}:=\left\{f_{n \pm k}^{(j)} \mid j, k \in \mathbb{N}_{0}\right\}$ is at most $m$-dimensional, any $m+1$ distinguished elements $g_{k}(k=0, \ldots, m)$ of $V_{f_{n}}$ are linearly dependent, i. e., an identity of the form

$$
\sum_{k=0}^{m} P_{k}(n, x) g_{k}(n, x)=0 \quad\left(P_{k} \in \mathbb{K}[n, x]\right)
$$

is valid. Given the defining holonomic recurrence equation and derivative rule, we can easily construct $P_{k}(k=0, \ldots, m)$ solving a linear system of equations, and therefore generate identity (23):

Algorithm 3. [Generate an identity] Let $f_{n}$ be an admissible family of order $m$, and $g_{k}(k=0, \ldots, m)$ denote $m+1$ distinguished elements of $V_{f_{n}}$. To generate an identity of the form (23),

1. Take (23) as an ansatz with still undetermined $P_{k}(k=0, \ldots, m)$.

2. Apply the derivative rule and the derivatives thereof to all $g_{k}$, recursively. This yields an equation

$$
\sum_{k=0}^{M} Q_{k}(n, x) h_{k}(n, x)=0
$$

with rational $Q_{k} \in \mathbb{K}(n, x)$ (depending on $P_{k}$ ) and $h_{k}=f_{n \pm n_{k}}$ that are shifts of $f_{n}$

3. Apply the recurrence equation recursively to (24) until only $m$ successive shifts of $f_{n}$ remain

4. Set the coefficient list of the system $h_{k}$ equal to zero, and solve the system for the $m+1$ indeterminates $P_{k}(k=0, \ldots, m)$.

5. Substituting $P_{k}(k=0, \ldots, m)$, and multiplying with the common denominator yields (23). 
Proof. Theorem 3 shows that a solution exists. By the described method obviously the sum (23) is represented by a linear combination of $m$ shifts of $f_{n}$. If its coefficients vanish, then the linear combination equals zero. The linear algebra technique described generates the values $P_{k}(k=0, \ldots, m)$ for which this is the case.

We note that the identity generated is unique if it is guaranteed that $f_{n}, f_{n-1}, \ldots$, $f_{n-m+1}$ are linearly independent over $\mathbb{K}(n, x)$, see the remark after the proof of Theorem 3.

\section{APPLICATION TO SPECTRAL APPROXIMATION}

In this section, we give an application of Algorithm 3 in the field of spectral approximation (see [6]). There, it is essential to have a family $f_{n}(x)$ of orthogonal polynomials possessing a representation

$$
f_{n}(x)=A_{n} f_{n-1}^{\prime}(x)+B_{n} f_{n+1}^{\prime}(x)
$$

in terms of the derivatives $f_{n \pm 1}^{\prime}$ with coefficients $A_{n}, B_{n}$ that are constant with respect to $x$.

In $([6], \S 2.3 .2)$, it is described how such an identity

$$
(2 n+1) P_{n}(x)=P_{n+1}^{\prime}(x)-P_{n-1}^{\prime}(x) \quad(n \in \mathbb{N})
$$

for the Legendre polynomials $f_{n}(x)=P_{n}(x)$ is applied.

Whereas our theory of admissible families guarantees the existence of a relation of type (25), namely a linear relation between $f_{n}(x), f_{n-1}^{\prime}(x)$, and $f_{n+1}^{\prime}(x)$ with polynomial coefficients in $x$ for any admissible family of order two, in particular for systems of orthogonal polynomials, the fact that the coefficients in (26) do not depend on $x$, is fortunate. This, in general, is not the case.

If we calculate the resulting relations for the Laguerre, Jacobi, Gegenbauer, Chebyshev and Hermite polynomials by Algorithm 3, we realize that mostly these are known formulas for those polynomials. We recall them here, omitting however the lengthy formula that we obtain for the Jacobi polynomials: For the generalized Laguerre polynomials $L_{n}^{(\alpha)}(x)$, we have

$$
(\alpha+2 n-x) L_{n}^{(\alpha)}(x)=-(\alpha+n) \frac{\partial}{\partial x} L_{n-1}^{(\alpha)}(x)+(\alpha+n-x) \frac{\partial}{\partial x} L_{n+1}^{(\alpha)}(x),
$$

for the Gegenbauer polynomials $C_{n}^{(\lambda)}(x)$, we have $(\lambda \neq 0)$

$$
2(\lambda+n) C_{n}^{(\lambda)}(x)=\frac{\partial}{\partial x} C_{n-1}^{(\lambda)}(x)-\frac{\partial}{\partial x} C_{n+1}^{(\lambda)}(x),
$$

for the Chebyshev polynomials $T_{n}(x)$, we have

$$
2\left(n^{2}-1\right) T_{n}(x)=(1+n) T_{n-1}^{\prime}(x)+(1-n) T_{n+1}^{\prime}(x),
$$

for the Chebyshev polynomials $U_{n}(x)$, we have

$$
2(1+n) U_{n}(x)=U_{n-1}^{\prime}(x)-U_{n+1}^{\prime}(x),
$$


and for the Hermite polynomials $H_{n}(x)$, we have

$$
2(1+n) H_{n}(x)=H_{n+1}^{\prime}(x)
$$

We see that in the case of the Gegenbauer, Chebyshev, and Hermite polynomials, the method succeeds, and these polynomials can be handled similarly to the Legendre polynomials. In the case of the Laguerre and Jacobi polynomials, however, the relations between $f_{n}(x), f_{n-1}^{\prime}(x)$, and $f_{n+1}^{\prime}(x)$ have coefficients depending explicitly on $x$, so we have to modify the method.

We notice that the given method can succeed even if a relation between the four terms $f_{n}(x), f_{n-1}^{\prime}(x), f_{n}^{\prime}(x)$, and $f_{n+1}^{\prime}(x)$ with coefficients not depending on $x$, exists. It turns out that such a formula exists for any of the classical families of nondiscrete polynomials. We saw already the results for the Gegenbauer, Chebyshev, and Hermite polynomials, and we will consider now the Laguerre and Jacobi polynomials. To obtain the announced relations, we use Algorithm 3 which generates a solution space of dimension one. If we are lucky, we can choose the free parameter such that, indeed, the coefficients occurring are independent of $x$.

Here are the results:

Theorem 6. For the generalized Laguerre polynomials $L_{n}^{(\alpha)}(x)$, the identity

$$
L_{n}^{(\alpha)}(x)=\frac{\partial}{\partial x} L_{n}^{(\alpha)}(x)-\frac{\partial}{\partial x} L_{n+1}^{(\alpha)}(x)
$$

is valid.

For the Jacobi polynomials $P_{n}^{(\alpha, \beta)}(x)$, the identity

$$
\begin{aligned}
P_{n}^{(\alpha, \beta)}(x)= & -\frac{2(\alpha+n)(\beta+n)}{(\alpha+\beta+n)(\alpha+\beta+2 n)(\alpha+\beta+2 n+1)} \frac{\partial}{\partial x} P_{n-1}^{(\alpha, \beta)}(x) \\
& +\frac{2(\alpha-\beta)}{(\alpha+\beta+2 n)(\alpha+\beta+2 n+2)} \frac{\partial}{\partial x} P_{n}^{(\alpha, \beta)}(x) \\
& +\frac{2(\alpha+\beta+n+1)}{(\alpha+\beta+2 n+1)(\alpha+\beta+2 n+2)} \frac{\partial}{\partial x} P_{n+1}^{(\alpha, \beta)}(x)
\end{aligned}
$$

is valid.

Note that (27) is well-known (see [34], VI (1.14)), whereas (29) is new. Note moreover that (29) shows that only in the case $\alpha=\beta$ a representation of the Jacobi polynomials $P_{n}^{(\alpha, \beta)}(x)$ in terms of only two of $\frac{\partial}{\partial x} P_{n-1}^{(\alpha, \beta)}(x), \frac{\partial}{\partial x} P_{n}^{(\alpha, \beta)}(x)$, or $\frac{\partial}{\partial x} P_{n+1}^{(\alpha, \beta)}(x)$ with coefficients independent of $x$ exists. For another method to deduce identity (29) and similar ones, see [18]. 


\section{IDENTIFICATION OF ADMISSIBLE FAMILIES}

In this section, we extend Zeilberger's holonomic approach [36] to identify holonornic families as admissible ones using Gröbner basis techniques.

Assume a holonomic family $f_{n}(x)$ is given by its holonomic differential equation with respect to $x$ and by its holonomic recurrence equation with respect to $n$. We write these equations in operator notation using the differential operator $D$ given by $D f_{n}(x)=f_{n}^{\prime}(x)$, and the (forward) shift operator $N$ given by $N f_{n}(x)=f_{n+1}(x)$. This procedure converts the two holonomic equations into a polynomial equations system in a noncommutative polynomial ring: From the product rule it follows that $D\left(x f_{n}(x)\right)-x D f_{n}(x)=f_{n}(x)$, and therefore we have the commutator relation $D x-x D=1$. On the other hand, for the shift operator we have $N\left(n f_{n}(x)\right)-n N f_{n}(x)=(n+1) f_{n+1}(x)-n f_{n+1}(x)=f_{n+1}(x)=N f_{n}(x)$, and therefore we have the commutator rule $N n-n N=N$.

As an example let us consider the Legendre polynomials $f_{n}(x)=P_{n}(x)$ : They form a holonomic family given by the holonomic equations

$$
\left(x^{2}-1\right) f_{n}^{\prime \prime}(x)+2 x f_{n}^{\prime}(x)-n(1+n) f_{n}(x)=0
$$

and

$$
(n+2) f_{n+2}(x)-(3+2 n) x f_{n+1}(x)+(n+1) f_{n}(x)=0
$$

(compare (2)-(3)), written here in terms of forward shifts.

In operator notation the holonomic equations read

$$
\left(x^{2}-1\right) D^{2}+2 x D-n(1+n)=0 \text { and }(n+2) N^{2}-(3+2 n) x N+(n+1)=0 .
$$

If we want to generate a derivative rule from (29), this is an elimination problem in the given noncommutative polynomial ring which can be solved by Gröbner basis methods ([5], [13], [36], [39], [31]-[33]).

The Gröbner basis of the left ideal generated by (29) with respect to the lexicographic term order $(D, N, n, x)$ is given by

$$
\begin{gathered}
\left\{\left(x^{2}-1\right) D^{2}+2 x D-n(1+n),(1+n) N D-(1+n) x D-(1+n)^{2}\right. \\
\left(x^{2}-1\right) N D-(1+n) x N+(1+n) \\
(1+n)\left(x^{2}-1\right) D-(1+n)^{2} N+x(1+n)^{2} \\
\left.(n+2) N^{2}-(3+2 n) x N+(n+1)\right\}
\end{gathered}
$$

we used the REDUCE implementation [24] (see [12]) for the noncommutative Gröbner calculations of this article. After the calculation of the Gröbner basis, for better readability the operators $D$ and $N$ were positioned back to the right, so that the equations can be easily understood as operator equations, again.

By the given term order, the Gröbner basis contains those equations for which the $D$-powers are eliminated furthest possible, and $(30)-(31)$ correspond to the relations

$$
\left(x^{2}-1\right) P_{n+1}^{\prime}(x)=(1+n)\left(x P_{n+1}(x)-P_{n}(x)\right),
$$




$$
\left(x^{2}-1\right) P_{n}^{\prime}(x)=(1+n)\left(P_{n+1}(x)-x P_{n}(x)\right)
$$

between the Legendre polynomials and their derivatives.

Therefore, we see that the calculation of the Gröbner basis in particular constructed the derivative rule for the Legendre polynomials, and therefore identified them as an admissible family.

The following algorithm generalizes this method for the general case:

Algorithm 4. Let $f_{n}(x)$ be a holonomic family given by the holonomic equations

$$
P(D, n, x) f_{n}(x)=0 \quad \text { and } \quad Q(N, n, x) f_{n}(x)=0
$$

in operator notation. Assume further that the holonomic equations are of lowest possible order. Then the following procedure determines whether or not $f_{n}(x)$ is an admissible family, and returns a forward derivative rule in the affirmative case.

1. Input: the holonomic representation of $f_{n}$ in form of the polynomials $P(D, n, x)$ and $Q(N, n, x)$.

2. Calculate the Gröbner basis $\mathcal{G}$ of the left ideal generated by $P$ and $Q$ with respect to the lexicographic term order $(D, N, n, x)$ by a noncommutative version of Buchberger's algorithm. The use of a weighted or graded order can simplify the procedure.

3. Choose the subset $\mathcal{L} \subset \mathcal{G}$ of polynomials that are linear with respect to $D$.

4. Check whether one of the polynomials in $\mathcal{L}$ contains a product of $D$ and $N$ or not. If yes, this is either the derivative rule $R(D, N, n, x)$; or the derivative rule $R(D, N, n, x)$ is easily constructed from the given polynomial, iteratively using the recurrence equation to decrease the order of $N$; go to (7.).

5. Iterate the following: Take the two polynomials $p_{1}, p_{2} \in \mathcal{L}$ having highest degree terms of the form $D N^{k}$. Make them of equal degree multiplying the lower order polynomial by a suitable power of $N$. Construct a polynomial $p_{3}$ as a linear combination such that the degree of the highest term $D N^{k}$ decreases. Replace $p_{1}$ and $p_{2}$ in $\mathcal{L}$ by $p_{3}$.

6. Check whether any $p \in \mathcal{L}$ contains products of $D$ and $N$ or not. If yes, this is either the derivative rule $R(D, N, n, x)$; or the derivative rule $R(D, N, n, x)$ is easily constructed from the given polynomial, iteratively using the recurrence equation to decrease the order of $N$; go to (7.). If not, the present method fails.

7. Output: the derivative rule $R(D, N, n, x)$.

Proof. Using the lexicographic term order $(D, N, n, x)$ the Gröbner basis $\mathcal{G}$ calculated in step (2.) eliminates $D$ to the lowest possible order. Therefore if the left ideal $\mathcal{I}$ generated by $P$ and $Q$ contains a forward derivative rule, $\mathcal{G}$ contains at least one element of degree one with respect to $D$. If such an element exists for which no term $D N$ occurs, then the procedure given in (4.) generates the derivative rule. Finally, the iteration in (5.) gets rid of highest powers of $D N^{k}$ (which may not 
occur with a suitable chosen graded or weighted order), and leads to the derivative rule, eventually, or the method fails.

Note, that the algorithm decides that no derivative rule exists for the Airy functions $f_{n}(x):=\mathrm{Ai}(x)$, given by (14) (under the hypothesis of the linear independence of $\mathrm{Ai}(x)$ and $\mathrm{Ai}^{\prime}(x)$ over $\left.\mathbb{K}(x)\right)$.

On the other hand, by the given method, it is possible to construct all derivative rules that can be found in [1] by the corresponding differential and recurrence equations besides one for the associated Legendre functions since in this case the recurrence equation does not have polynomial coefficients. Note that we always used a weighted lexicographic order with weights $(2,1,0,0)$ and never entered parts (5.) and (6.) of Algorithm 4

The most time consuming results in this direction are the calculation of the derivative rules for the Jacobi polynomials $P_{n}^{(\alpha, \beta)}(x)$, see Theorem 8 , since this is a 6 -variable problem. Note that, in a similar treatment, Chyzak tried to derive this type of result with a MAPLE implementation, without success ([7], §4.1). Using the REDUCE implementation [24], any of these calculations (for the Jacobi polynomials) needs about five minutes on a DEC Alpha workstation (using the ezgcd switch, see [12], §9.3). Note that also with a pure lexicographic term order, the Gröbner bases are derived in a similar time. For all other families considered, the calculation needs only seconds

As an example, we consider the Whittaker functions $M_{n, m}(x)$ with respect to the parameter $m$ for which no derivative rule is listed in [1], see $\S 13.4$. In Theorem 1, we found the holonomic recurrence equation

$$
\begin{aligned}
M_{n, m+1}(x)= & 16 \frac{(1+2 m) m\left(4 m^{2}-1-2 n x\right)(m+1)}{x(2 m-1)(2 n+1+2 m)(2 n-1-2 m)} M_{n, m}(x) \\
& -16 \frac{(m+1) m(1+2 m)^{2}}{(2 n+1+2 m)(2 n-1-2 m)} M_{n, m-1}(x) .
\end{aligned}
$$

On the other hand, the holonomic differential equation

$$
\left(1-4 m^{2}+4 n x-x^{2}\right) M_{n, m}(x)+4 x^{2} M_{n, m}^{\prime \prime}(x)=0
$$

is well-known ([1], (13.1.31)). Therefore we have the operator polynomials

$$
P(D, m, x):=1-4 m^{2}+4 n x-x^{2}+4 x^{2} D^{2},
$$

and

$$
\begin{aligned}
Q(M, m, x):= & 16(1+m)(2+m)(1+2 m)(3+2 m)^{2} x \\
& +16(1+m)(2+m)(3+2 m)\left(2 n x-3-8 m-4 m^{2}\right) M \\
& +(1+2 m)(2 n-3-2 m)(3+2 m+2 n) x M^{2},
\end{aligned}
$$

$M$ denoting the shift operator with respect to $m$.

Using the method described above to eliminate the second order terms of $D$ and $M$, we find for the Whittaker functions: 
Theorem 7. The Whittaker function $M_{n, m}(x)$ satisfy the forward and backward derivative rules

$$
\begin{aligned}
M_{n, m}^{\prime}(x)= & \frac{\left(1+4 m+4 m^{2}-2 n x\right)}{2(1+2 m) x} M_{n, m}(x) \\
& +\frac{(1+2 m-2 n)(1+2 m+2 n)}{8(1+m)(1+2 m)^{2}} M_{n, m+1}(x)
\end{aligned}
$$

and

$$
M_{n, m}^{\prime}(x)=2 m M_{n, m-1}(x)+\frac{\left(-1+4 m-4 m^{2}+2 n x\right)}{2(-1+2 m) x} M_{n, m}(x)
$$

with respect to $m$.

Similarly, the Whittaker functions $W_{n, m}(x)$ satisfy the forward and backward derivative rules

$$
W_{n, m}^{\prime}(x)=\frac{\left(1+4 m+4 m^{2}-2 n x\right)}{2(1+2 m) x} W_{n, m}(x)+\frac{(-1-2 m+2 n)}{2(1+2 m)} W_{n, m+1}(x)
$$

and

$$
W_{n, m}^{\prime}(x)=\frac{(-1+2 m+2 n)}{2(1-2 m)} W_{n, m-1}(x)+\frac{\left(-1+4 m-4 m^{2}+2 n x\right)}{2(-1+2 m) x} W_{n, m}(x) .
$$

Note that $M_{n, m}(x)$ is one of the rare cases for which the second part of part (4.) of Algorithm 4 is entered.

In the rest of this section, we apply Algorithm 4 to derive derivative rules for the classical orthogonal families. Well-known are backward or forward derivative representations for the derivative with respect to $x$ of the (nondiscrete) orthogonal polynomials $f_{n}(x)$ in terms of $f_{n}(x)$ and $f_{n-1}(x)$, or in terms of $f_{n}(x)$ and $f_{n+1}(x)$.

Note that derivative rules with respect to $n$ are commonly stated (see [1], (22.8)), whereas those with respect to the other parameters are not.

Applying the above mentioned algorithms to the generalized Laguerre, Jacobi, and Gegenbauer polynomials yields the following derivative rules:

Theorem 8. The generalized Laguerre polynomials $L_{n}^{(\alpha)}(x)$ satisfy the forward and backward derivative rules

$$
\begin{aligned}
\frac{\partial}{\partial x} L_{n}^{(\alpha)}(x) & =\frac{-1-\alpha-n+x}{x} L_{n}^{(\alpha)}(x)+\frac{1+n}{x} L_{n+1}^{(\alpha)}(x) \\
& =-\frac{\alpha+n}{x} L_{n-1}^{(\alpha)}(x)+\frac{n}{x} L_{n}^{(\alpha)}(x)=L_{n}^{(\alpha)}(x)-L_{n}^{(\alpha+1)}(x) \\
& =\frac{\alpha+n}{x} L_{n}^{(\alpha-1)}(x)-\frac{\alpha}{x} L_{n}^{(\alpha)}(x)
\end{aligned}
$$

with respect to the parameters $n$, and $\alpha$. 
The Jacobi polynomials $P_{n}^{(\alpha, \beta)}(x)$ satisfy the forward and backward derivative rules

$$
\begin{aligned}
\frac{\partial}{\partial x} P_{n}^{(\alpha, \beta)}(x)=\frac{(1+\alpha+\beta+n)(\alpha-\beta+(2+\alpha+\beta+2 n) x}{(2+\alpha+\beta+2 n)\left(1-x^{2}\right)} P_{n}^{(\alpha, \beta)}(x) & \\
& -\frac{2(1+n)(1+\alpha+\beta+n)}{(2+\alpha+\beta+2 n)\left(1-x^{2}\right)} P_{n+1}^{(\alpha, \beta)}(x) \\
= & \frac{2(\alpha+n)(\beta+n)}{(\alpha+\beta+2 n)\left(1-x^{2}\right)} P_{n-1}^{(\alpha, \beta)}(x)-\frac{n(-\alpha+\beta+\alpha x+\beta x+2 n x)}{(\alpha+\beta+2 n)\left(1-x^{2}\right)} P_{n}^{(\alpha, \beta)}(x) \\
= & -\frac{1+\alpha+\beta+n}{1+x} P_{n}^{(\alpha, \beta)}(x)+\frac{1+\alpha+\beta+n}{1+x} P_{n}^{(\alpha+1, \beta)}(x) \\
= & -\frac{2(\alpha+n)}{1-x^{2}} P_{n}^{(\alpha-1, \beta)}(x)+\frac{2 \alpha+n-n x}{1-x^{2}} P_{n}^{(\alpha, \beta)}(x) \\
= & \frac{1+\alpha+\beta+n}{1-x} P_{n}^{(\alpha, \beta)}(x)-\frac{1+\alpha+\beta+n}{1-x} P_{n}^{(\alpha, \beta+1)}(x) \\
= & \frac{2(\beta+n)}{1-x^{2}} P_{n}^{(\alpha, \beta-1)}(x)-\frac{2 \beta+n+n x}{1-x^{2}} P_{n}^{(\alpha, \beta)}(x)
\end{aligned}
$$

with respect to the parameters $n, \alpha$, and $\beta$.

The Gegenbauer polynomials $C_{n}^{(\lambda)}(x)$ satisfy the forward and backward derivative rules

$$
\begin{aligned}
\frac{\partial}{\partial x} C_{n}^{(\lambda)}(x) & =\frac{(2 \lambda+n) x}{1-x^{2}} C_{n}^{(\lambda)}(x)-\frac{1+n}{1-x^{2}} C_{n+1}^{(\lambda)}(x) \\
& =-\frac{1-2 \lambda-n}{1-x^{2}} C_{n-1}^{(\lambda)}(x)-\frac{n x}{1-x^{2}} C_{n}^{(\lambda)}(x) \\
& =-\frac{2 \lambda+n}{x} C_{n}^{(\lambda)}(x)+\frac{2 \lambda}{x} C_{n}^{(\lambda+1)}(x) \\
& =-\frac{(-2+2 \lambda+n)(-1+2 \lambda+n)}{2(\lambda-1) x\left(1-x^{2}\right)} C_{n}^{(\lambda-1)}(x)-\frac{1-2 \lambda-n+n x^{2}}{x\left(1-x^{2}\right)} C_{n}^{(\lambda)}(x)
\end{aligned}
$$

with respect to the parameters $n$, and $\lambda$.

Similarly, we get for the Krawtchouk, Meixner, discrete Laguerre, and Charlier polynomials the following derivative representations.

Theorem 9. The Krawtchouk polynomials $k_{n}^{(p)}(x, N)$ satisfy the forward and backward derivative rules

$$
\frac{\partial}{\partial p} k_{n}^{(p)}(x, N)=\frac{-n+2 n p-N p+x}{(p-1) p} k_{n}^{(p)}(x, N)+\frac{1+n}{(1-p) p} k_{n+1}^{(p)}(x, N)
$$




$$
\begin{aligned}
& =(-1+n-N) k_{n-1}^{(p)}(x, N) \\
& =\frac{n-N+x}{p-1} k_{n}^{(p)}(x, N)+\frac{N-x}{p-1} k_{n}^{(p)}(x+1, N) \\
& =\frac{x}{p} k_{n}^{(p)}(x-1, N)+\frac{n-x}{p} k_{n}^{(p)}(x, N) \\
& =\frac{-1+n-N}{p} k_{n}^{(p)}(x, N)+\frac{1-n+N}{p} k_{n}^{(p)}(x, N+1) \\
& =\frac{N-x}{(p-1) p} k_{n}^{(p)}(x, N-1)+\frac{-N+n p+x}{(p-1) p} k_{n}^{(p)}(x, N)
\end{aligned}
$$

with respect to the parameters $n, x$, and $N$.

The Meixner polynomials $m_{n}^{(\gamma, \mu)}(x)$ satisfy the forward and backward derivative rules

$$
\begin{aligned}
\frac{\partial}{\partial \mu} m_{n}^{(\gamma, \mu)}(x) & =\frac{\gamma \mu+\mu n-x+\mu x}{(1-\mu) \mu} m_{n}^{(\gamma, \mu)}(x)-\frac{1}{1-\mu} m_{n+1}^{(\gamma, \mu)}(x) \\
& =\frac{n(-1+\gamma+n)}{(1-\mu) \mu} m_{n-1}^{(\gamma, \mu)}(x)-\frac{n}{(1-\mu) \mu} m_{n}^{(\gamma, \mu)}(x) \\
& =-\frac{\gamma+n+x}{\mu} m_{n}^{(\gamma, \mu)}(x)+\frac{\gamma+x}{\mu} m_{n}^{(\gamma+1, \mu)}(x) \\
& =\frac{(-1+\gamma+n)}{\mu-1} m_{n}^{(\gamma-1, \mu)}(x)+\frac{(1-\gamma)}{(\mu-1) \mu} m_{n}^{(\gamma, \mu)}(x) \\
& =\frac{\gamma \mu-n+\mu n+\mu x}{(1-\mu) \mu} m_{n}^{(\gamma, \mu)}(x)-\frac{\gamma+x}{1-\mu} m_{n}^{(\gamma, \mu)}(x+1) \\
& =\frac{x}{(1-\mu) \mu} m_{n}^{(\gamma, \mu)}(x-1)-\frac{x}{(1-\mu) \mu} m_{n}^{(\gamma, \mu)}(x)
\end{aligned}
$$

with respect to the parameters $n, \gamma$, and $x$.

The discrete Laguerre polynomials $l_{n}^{(\rho, \alpha)}(x)$ satisfy the forward and backward derivative rules

$$
\begin{aligned}
\frac{\partial}{\partial \rho} l_{n}^{(\rho, \alpha)}(x) & =\frac{-1-n-\alpha \rho+x-\rho x}{(\rho-1) \rho} l_{n}^{(\rho, \alpha)}(x)+\frac{1+n}{(\rho-1) \rho} l_{n+1}^{(\rho, \alpha)}(x) \\
& =\frac{\alpha+n}{1-\rho} l_{n-1}^{(\rho, \alpha)}(x)-\frac{n}{1-\rho} l_{n}^{(\rho, \alpha)}(x) \\
& =-\frac{\alpha+x}{\rho} l_{n}^{(\rho, \alpha)}(x)+\frac{\alpha+x}{\rho} l_{n}^{(\rho, \alpha+1)}(x) \\
& =\frac{\alpha+n}{(\rho-1) \rho} l_{n}^{(\rho, \alpha-1)}(x)+\frac{-\alpha-n+n \rho}{(\rho-1) \rho} l_{n}^{(\rho, \alpha)}(x)
\end{aligned}
$$




$$
\begin{aligned}
& =\frac{\alpha+x}{1-\rho} l_{n}^{(\rho, \alpha)}(x)-\frac{\alpha+x}{1-\rho} l_{n}^{(\rho, \alpha)}(x+1) \\
& =\frac{1-x}{(\rho-1) \rho} l_{n}^{(\rho, \alpha)}(x-1)+\frac{-1-n+n \rho+x}{(\rho-1) \rho} l_{n}^{(\rho, \alpha)}(x)
\end{aligned}
$$

with respect to the parameters $n, \alpha$, and $x$.

The Charlier polynomials $c_{n}^{(\mu)}(x)$ satisfy the forward and backward derivative rules

$$
\begin{aligned}
\frac{\partial}{\partial \mu} c_{n}^{(\mu)}(x) & =\frac{\mu-x}{\mu} c_{n}^{(\mu)}(x)-c_{n+1}^{(\mu)}(x) \\
& =\frac{n}{\mu} c_{n-1}^{(\mu)}(x)-\frac{n}{\mu} c_{n}^{(\mu)}(x) \\
& =\frac{\mu-n}{\mu} c_{n}^{(\mu)}(x)-c_{n}^{(\mu)}(x+1) \\
& =\frac{x}{\mu} c_{n}^{(\mu)}(x-1)-\frac{x}{\mu} c_{n}^{(\mu)}(x)
\end{aligned}
$$

with respect to the parameters $n$, and $x$.

Note that many of the above derivative rules are a direct consequence of Theorem 5 in view of the hypergeometric representations (7)-(11).

Note further that some of the above derivative rules have appeared in the literature. Relation (32) can be found in $([8],(3.19))$, for $\alpha=0$, and in $([35],(2.28))$ for general $\alpha$, and (33) is in ([8], (3.31)). However, these relations did not appear systematically anywhere in the literature. All given representations can be calculated by a computer algebra system, and were done by the REDUCE implementation [24], as well as by our implementation in Mathematica [17].

This section can be summarized as follows: If a holonomic family $f_{\mathfrak{n}}(x)$ is given by a holonomic differential and a holonomic recurrence equation, it would be wise to use Algorithm 4 to find a derivative rule for $f_{n}(x)$. In the affirmative case, any identity of the form of Algorithm 3 can be discovered by linear algebra techniques only. So only in the first step, Gröbner basis techniques are needed to discover whether or not $f_{n}(x)$ forms an admissible family.

\section{REPRESENTATIONS OF PARAMETER DERIVATIVES}

Whereas, for any of the classical nondiscrete families $f_{n}(x)$ of orthogonal polynomials a representation of the derivative with respect to the principal nondiscrete variable $x$ in terms of $f_{n}(x)$ and $f_{n-1}(x)$ exists (see [34], Chapter IV, (4.8)), this is, in general, not the case with respect to other variables involved. Fröhlich [11] gives, however, the following argument: For any family $f_{n}^{(\alpha)}(x)=\sum_{k=0}^{n} a_{k}(\alpha) x^{k}$ of orthogonal polynomials there is a representation of the form

$$
\frac{\partial}{\partial \alpha} f_{n}^{(\alpha)}(x)=\sum_{k=0}^{n} c_{k}^{(\alpha)} f_{k}^{(\alpha)}(x)
$$


for the $\alpha$-derivative of $f_{n}^{(\alpha)}(x)$, since by termwise differentiation, the expression

$$
\frac{\partial}{\partial \alpha} f_{n}^{(\alpha)}(x)=\sum_{k=0}^{n} \frac{\partial}{\partial \alpha} a_{k}(\alpha) x^{k}
$$

is seen to be a polynomial of degree $n$ with respect to $x$, and since any polynomial of degree $n$ has a representation of the form (34) by the orthogonality of the family $f_{n}^{(\alpha)}(x)$. Rather than having representations in terms of the last two polynomials, in the given situation we generally have representations in terms of the complete system $\left\{f_{k}^{(\alpha)}(x) \mid k=0, \ldots, n\right\}$. We call the derivative with respect to $\alpha$ a parameter derivative of $f_{n}^{(\alpha)}(x)$.

It is a simple task to give representations of the parameter derivatives for hypergeometric functions, and families of orthogonal polynomials in terms of symbolic sums of hypergeometric functions, or in terms of the $\psi$-function (see [1], $\S 6.3$ ), by termwise differentiation of the defining series representations, since

$$
\begin{aligned}
\frac{\frac{\partial}{\partial \alpha}(\alpha)_{n}}{(\alpha)_{n}} & =\frac{\partial}{\partial \alpha}\left(\ln (\alpha)_{n}\right)=\frac{\partial}{\partial \alpha}\left(\ln \prod_{k=0}^{n-1}(\alpha+k)\right) \\
& =\frac{\partial}{\partial \alpha} \sum_{k=0}^{n-1} \ln (\alpha+k)=\sum_{k=0}^{n-1} \frac{1}{\alpha+k}=\psi(\alpha+n)-\psi(\alpha) .
\end{aligned}
$$

On the other hand, generally it is a nontrivial question to determine the coefficients $c_{k}^{(\alpha)}$ of representation (34).

In connection with the development of Galerkin methods, i. e. numerical techniques involving orthogonal polynomials, a result in this direction was given by Wulkow who obtained the parameter derivative representation

$$
\frac{\partial}{\partial \alpha} l_{n}^{(\rho, \alpha)}(x)=\sum_{k=0}^{n-1} \frac{\rho^{n-k}}{n-k} l_{k}^{(\rho, \alpha)}(x)
$$

([35], (2.30)) for the discrete Laguerre polynomials with respect to the parameter $\alpha$.

Using the hypergeometric representation (10), this identity can be rewritten

$$
\frac{\partial}{\partial c}\left(\frac{(c)_{n}}{n !}{ }_{2} F_{1}\left(\begin{array}{cc|c}
-n, & b & x \\
c & & x
\end{array}\right)\right)=\sum_{k=0}^{n-1} \frac{(c)_{k}}{k !(n-k)}{ }_{2} F_{1}\left(\begin{array}{cc|c}
-k, & b \\
c & x
\end{array}\right)
$$

as representation for the derivative of the Gauß hypergeometric polynomial with respect to its third argument $c$.

By the product rule, $(u v)^{\prime}=u^{\prime} v+v^{\prime} u$, i. e., $v^{\prime}=\frac{(u v)^{\prime}}{u}-\frac{u^{\prime}}{u} v$, and therefore we 
get from (37), using (36)

$$
\begin{gathered}
\frac{\partial}{\partial c}{ }_{2} F_{1}\left(\begin{array}{c}
-n, b \\
c
\end{array} \mid x\right)=\frac{n !}{(c)_{n}} \sum_{k=0}^{n-1} \frac{(c)_{k}}{k !(n-k)}{ }_{2} F_{1}\left(\begin{array}{c}
-k, b \\
c
\end{array} \mid x\right)-\frac{\frac{\partial}{\partial c}(c)_{n}}{(c)_{n}}{ }_{2} F_{1}\left(\begin{array}{c}
-n, b \\
c
\end{array} \mid x\right) \\
=\sum_{k=0}^{n-1}\left(\frac{-1}{c+k}{ }_{2} F_{1}\left(\begin{array}{c}
-n, b \\
c
\end{array} \mid x\right)+\frac{n !}{k !(n-k)(c+k)_{n-k}}{ }_{2} F_{1}\left(\begin{array}{c}
-k, b \\
c
\end{array}\right)\right) .
\end{gathered}
$$

Recently, Fröhlich deduced the analogous representation

$$
\begin{aligned}
& \frac{\partial}{\partial b}{ }_{2} F_{1}\left(\begin{array}{c|c}
-n, b & x \\
c &
\end{array}\right) \\
& =\sum_{k=0}^{n-1}\left(\frac{1}{b+k}{ }_{2} F_{1}\left(\begin{array}{c}
r \\
-n, b \\
c
\end{array}\right)-\frac{n !}{k !(n-k)(b+k)_{n-k}}{ }_{2} F_{1}\left(\begin{array}{c}
-k, b \\
c
\end{array}\right)\right)
\end{aligned}
$$

([11], Theorem 1) for the parameter derivative of the Gauß hypergeometric polynomial with respect to its second argument $b$.

By the product rule, again, we obtain from (36) and (39)

$$
\begin{aligned}
& \frac{\partial}{\partial b}\left(\frac{(b)_{n}}{n !}{ }_{2} F_{1}\left(\begin{array}{c|c}
-n, b & x)) \\
c &
\end{array}\right)\right. \\
= & \sum_{k=0}^{n-1}\left(\frac{2(b)_{n}}{(b+k) n !}{ }_{2} F_{1}\left(\begin{array}{c}
-n, b \\
c
\end{array} \mid x\right)-\frac{(b)_{k}}{k !(n-k)}{ }_{2} F_{1}\left(\begin{array}{c}
-k, b \\
c
\end{array} \mid x\right)\right)
\end{aligned}
$$

which is a statement similar to (37) for the parameter $b$. Note that the advantage of representations (36), and (37), is the fact that in these cases the derivative polynomials are of degree $n-1$, i. e. $c_{n}^{(\alpha)} \equiv 0$, and therefore the $n$th polynomial $f_{n}^{(\alpha)}(x)$ does not explicitly appear on the right hand side. Whether this situation applies or not, depends on the standardization that is used. Further, we realize that in (36), and (37), the $\psi$-function does not occur on the right hand side, either. Unfortunately, the other derivative representations mentioned do not have the same simple structure.

Using (39), Fröhlich moreover obtained the following representations of the parameter derivatives

$$
\begin{aligned}
\frac{\partial}{\partial \alpha} P_{n}^{(\alpha, \beta)}(x)= & \sum_{k=0}^{n-1} \frac{1}{\alpha+\beta+1+k+n} \\
& \times\left(P_{n}^{(\alpha, \beta)}(x)+\frac{\alpha+\beta+1+2 k}{n-k} \frac{(\beta+k+1)_{n-k}}{(\alpha+\beta+k+1)_{n-k}} P_{k}^{(\alpha, \beta)}(x)\right),
\end{aligned}
$$


and

$$
\begin{aligned}
\frac{\partial}{\partial \beta} P_{n}^{(\alpha, \beta)}(x) & =\sum_{k=0}^{n-1} \frac{1}{\alpha+\beta+1+k+n} \\
& \times\left(P_{n}^{(\alpha, \beta)}(x)+(-1)^{n-k} \frac{\alpha+\beta+1+2 k}{n-k} \frac{(\alpha+k+1)_{n-k}}{(\alpha+\beta+k+1)_{n-k}} P_{k}^{(\alpha, \beta)}(x)\right)
\end{aligned}
$$

([11], Theorem 3) for the Jacobi polynomials $P_{n}^{(\alpha, \beta)}(x)$ with respect to $\alpha$ and $\beta$.

Note that these results implicitly are also contained in work of Gábor Szegö ([30], (9.4.4), compare [2], (13), and [3], (2.7)-(2.8))

$$
\begin{aligned}
P_{n}^{(a, \beta)}(x)= & \sum_{k=0}^{n} \frac{\Gamma(n+\beta+1)(2 k+\alpha+\beta+1)}{\Gamma(a-\alpha) \Gamma(n+a+\beta+1)} \\
& \times \frac{\Gamma(n+k+a+\beta+1) \Gamma(n-k+a-\alpha) \Gamma(k+\alpha+\beta+1)}{\Gamma(n+k+\alpha+\beta+2) \Gamma(n-k+1) \Gamma(k+\beta+1)} P_{k}^{(\alpha, \beta)}(x) .
\end{aligned}
$$

The limit $a \rightarrow \alpha$ yields the first result; similarly the other case can be treated.

In the following theorem, we list some more parameter derivative representations for families of orthogonal polynomials, that can be obtained from the above results.

Theorem 10. For the generalized Laguerre polynomials $L_{n}^{(\alpha)}(x)$, we have the representation of the parameter derivative

$$
\frac{\partial}{\partial \alpha} L_{n}^{(\alpha)}(x)=\sum_{k=0}^{n-1} \frac{1}{n-k} L_{k}^{(\alpha)}(x)=\sum_{k=1}^{n} \frac{1}{k} L_{n-k}^{(\alpha)}(x)
$$

with respect to the parameter $\alpha$.

For the Gegenbauer polynomials $C_{n}^{(\lambda)}(x)$, we have the representation of the parameter derivative

$$
\begin{aligned}
\frac{\partial}{\partial \lambda} C_{n}^{(\lambda)}(x)= & \sum_{k=0}^{n-1}\left(\frac{2(1+k)}{(2 \lambda+k)(2 \lambda+1+2 k)}+\frac{2}{2 \lambda+k+n}\right) C_{n}^{(\lambda)}(x) \\
& +\sum_{k=0}^{n-1} \frac{2\left(1+(-1)^{n-k}\right)(\lambda+k)}{(2 \lambda+k+n)(n-k)} C_{k}^{(\lambda)}(x)
\end{aligned}
$$

with respect to the parameter $\lambda$.

For the Krawtchouk polynomials $k_{n}^{(p)}(x, N)$, we have the representation of the 
parameter derivative

$\frac{\partial}{\partial x} k_{n}^{(p)}(x, N)=\sum_{k=0}^{n-1}\left(\frac{1}{x-k} k_{n}^{(p)}(x, N)+\frac{(-1)^{n-k} p^{n-k}(N-n+1)_{n-k}}{(n-k)(k-x)_{n-k}} k_{k}^{(p)}(x, N)\right)$

with respect to the parameter $x$.

For the Meixner polynomials $m_{n}^{(\gamma, \mu)}(x)$, we have the representation of the parameter derivative

$$
\frac{\partial}{\partial \gamma} m_{n}^{(\gamma, \mu)}(x)=\sum_{k=0}^{n-1} \frac{n !}{k !(n-k)} m_{k}^{(\gamma, \mu)}(x)
$$

with respect to the parameter $\gamma$.

Proof. From (39), representation (41) easily follows using the limit relation

$$
L_{n}^{(\alpha)}(x)=\lim _{\beta \rightarrow \infty} P_{n}^{(\alpha, \beta)}\left(1-\frac{2 x}{\beta}\right)
$$

(see [1], (22.15.5)).

To deduce (43), we utilize the representation

$$
C_{n}^{(\lambda)}(x)=\frac{(2 \lambda)_{n}}{(\lambda+1 / 2)_{n}} P_{n}^{(\lambda-1 / 2, \lambda-1 / 2)}(x)
$$

(see [30], (4.7.1), and [34], V (7.2)) for the Gegenbauer polynomials, and use both, (39), and (40). Writing $\alpha=\lambda-1 / 2$, and $\beta=\lambda-1 / 2$, we get with the multidimensional chain rule

$$
\begin{aligned}
\frac{\partial}{\partial \lambda} C_{n}^{(\lambda)}(x)= & \frac{\partial}{\partial \lambda}\left(\frac{(2 \lambda)_{n}}{(\lambda+1 / 2)_{n}} P_{n}^{(\lambda-1 / 2, \lambda-1 / 2)}(x)\right) \\
= & \frac{1}{(\lambda+1 / 2)_{n}} P_{n}^{(\lambda-1 / 2, \lambda-1 / 2)}(x) \frac{\partial}{\partial \lambda}\left((2 \lambda)_{n}\right) \\
& +(2 \lambda)_{n} P_{n}^{(\lambda-1 / 2, \lambda-1 / 2)}(x) \frac{\partial}{\partial \lambda}\left(\frac{1}{(\lambda+1 / 2)_{n}}\right) \\
& +\frac{(2 \lambda)_{n}}{(\lambda+1 / 2)_{n}} \frac{\partial}{\partial \lambda}\left(P_{n}^{(\lambda-1 / 2, \lambda-1 / 2)}(x)\right) \\
= & \sum_{k=0}^{n-1}\left(\frac{2}{2 \lambda+k}-\frac{1}{\lambda+1 / 2+k}\right) C_{n}^{(\lambda)}(x) \\
& +\left.\frac{(2 \lambda)_{n}}{(\lambda+1 / 2)_{n}}\left(\frac{\partial}{\partial \alpha} P_{n}^{(\alpha, \beta)}(x)+\frac{\partial}{\partial \beta} P_{n}^{(\alpha, \beta)}(x)\right)\right|_{\alpha=\beta=\lambda-1 / 2} \\
= & \sum_{k=0}^{n-1}\left(\frac{2(1+k)}{(2 \lambda+k)(2 \lambda+1+2 k)}+\frac{2}{2 \lambda+k+n}\right) C_{n}^{(\lambda)}(x)
\end{aligned}
$$




$$
\begin{aligned}
& +\sum_{k=0}^{n-1}\left(\frac{1+(-1)^{n-k}}{2 \lambda+k+n} \frac{(2 \lambda)_{n}}{(\lambda+1 / 2)_{n}} \frac{2 \lambda+2 k}{n-k}\right. \\
& \times \frac{(\lambda+k+1 / 2)_{n-k}}{(2 \lambda+k)_{n-k}} P_{k}^{(\lambda-1 / 2, \lambda-1 / 2)}(x) \\
= & \sum_{k=0}^{n-1}\left(\frac{2(1+k)}{(2 \lambda+k)(2 \lambda+1+2 k)}+\frac{2}{2 \lambda+k+n}\right) C_{n}^{(\lambda)}(x) \\
& +\sum_{k=0}^{n-1} \frac{2\left(1+(-1)^{n-k}\right)(\lambda+k)}{(2 \lambda+k+n)(n-k)} C_{k}^{(\lambda)}(x),
\end{aligned}
$$

i. e. (43).

According to (7), representation (43) is a reformulation of (39), whereas relation (44) immediately follows from (36), using (10).

Using the abbreviation

$$
\begin{aligned}
g_{n}^{(\lambda)}(x) & =\sum_{k=0}^{n-1}\left(\frac{2(1+k)}{(2 \lambda+k)(2 \lambda+1+2 k)}+\frac{2}{2 \lambda+k+n}\right) C_{n}^{(\lambda)}(x) \\
& =\sum_{k=0}^{n-1}\left(\frac{2\left(2 k+3 k^{2}+4 \lambda+8 k \lambda+4 \lambda^{2}+n+k n\right)}{(2 \lambda+k)(2 \lambda+1+2 k)(2 \lambda+k+n)}\right) C_{n}^{(\lambda)}(x)
\end{aligned}
$$

representation (43) for the parameter derivative of $C_{n}^{(\lambda)}(x)$ can be rewritten as

$$
\begin{aligned}
\frac{\partial}{\partial \lambda} C_{n}^{(\lambda)}(x) & =g_{n}^{(\lambda)}(x)+\sum_{k=0}^{n-1} \frac{2\left(1+(-1)^{n-k}\right)(\lambda+k)}{(2 \lambda+k+n)(n-k)} C_{k}^{(\lambda)}(x) \\
& =g_{n}^{(\lambda)}(x)+\sum_{l=1}^{n} \frac{2\left(1+(-1)^{l}\right)(\lambda+n-l)}{(2 \lambda+2 n-l) l} C_{n-l}^{(\lambda)}(x) \\
& =g_{n}^{(\lambda)}(x)+\sum_{k=1}^{[n / 2]} \frac{\lambda+n-2 k}{(\lambda+n-k) k} C_{n-2 k}^{(\lambda)}(x) .
\end{aligned}
$$

Whereas (39)-(40) are rather difficult formulas, and difficult to obtain, formula (41) is so simple that it seems to be rather unlikely that it should not be found somewhere in the literature. Nevertheless, we were not successful doing so, hence (41) seems to be new. Note that it can also be deduced from ([2], (8)).

Furthermore, after having found this formula, it may be proved by other means as well. By the computations

$$
\begin{aligned}
\frac{\partial}{\partial \alpha} L_{n}^{(\alpha)}(x) & =\frac{\partial}{\partial \alpha}\left(\frac{(\alpha+1)_{n}}{n !} \sum_{j=0}^{n} \frac{(-n)_{j}}{(\alpha+1)_{j} j !} x^{j}\right) \\
& =\sum_{j=0}^{n} \frac{(-n)_{j}}{n ! j !} \frac{\partial}{\partial \alpha}\left((\alpha+1+j)_{n-j}\right) x^{j}
\end{aligned}
$$




$$
=\sum_{j=0}^{n-1} \frac{(-n)_{j}(\alpha+1+j)_{n-j}}{n ! j !} \sum_{k=0}^{n-j-1} \frac{1}{\alpha+1+j+k} x^{j},
$$

and

$$
\begin{aligned}
\sum_{k=0}^{n-1} \frac{1}{n-k} L_{k}^{(\alpha)}(x) & =\sum_{k=0}^{n-1} \frac{1}{n-k} \frac{(\alpha+1)_{k}}{k !} \sum_{j=0}^{k} \frac{(-k)_{j}}{(\alpha+1)_{j} j !} x^{j} \\
& =\sum_{j=0}^{n-1} \sum_{k=j}^{n-1} \frac{1}{n-k} \frac{(-k)_{j}}{k ! j !}(\alpha+1+j)_{k-j} x^{j}
\end{aligned}
$$

we may compare coefficients to find that (41) is equivalent to the identity $(j=$ $0, \ldots, n-1)$

$$
\frac{(-n)_{j}(\alpha+1+j)_{n-j}}{n ! j !} \sum_{k=0}^{n-j-1} \frac{1}{\alpha+1+j+k}=\sum_{k=j}^{n-1} \frac{1}{n-k} \frac{(-k)_{j}}{k ! j !}(\alpha+1+j)_{k-j},
$$

or equivalently

$$
\begin{aligned}
a(n, j) & =\sum_{k=0}^{n-j-1} \frac{1}{\alpha+1+j+k}=\sum_{k=j}^{n-1} \frac{n !}{(n-k) k !} \frac{(-k)_{j}}{(-n)_{j}} \frac{(\alpha+1+j)_{k-j}}{(\alpha+1+j)_{n-j}} \\
& =\sum_{k=j}^{n-1} \frac{n !}{(n-k) k !} \frac{(k-j+1)_{j}}{(n-j+1)_{j}} \frac{1}{(\alpha+1+k)_{n-k}}=A(n, j) .
\end{aligned}
$$

The last identity can easily be proved by Zeilberger's algorithm: the result is that both sides of (46) satisfy the inhomogeneous first order recurrence equation (see [26])

$$
a(n+1, j)-a(n, j)=A(n+1, j)-A(n, j)=\frac{1}{1+\alpha+n}
$$

of the $\psi$-function, having the same initial value $a(j+1, j)=A(j+1, j)=\frac{1}{\alpha+1+j}$.

\section{ACKNOWLEDGEMENTS}

I would like to thank Prof. Peter Deufhard for his support, and encouragement to work on the given subject, and for his valuable comments on the topic. Moreover many discussions with Jochen Fröhlich were very helpful. In particular, I am indebted to him to bring the problem of Section 7 to my attention. Finally, I thank Herbert Melenk for his advice on Gröbner bases, and his support with respect to the REDUCE implementation [24]. 


\section{REFERENCES}

1. M. Abramowitz and I.A. Stegun, Handbook of Mathematical Functions. Dover Publ., New York, 1964.

2. R. Askey, Dual equations and classical orthogonal polynomials, J. Math. Anal. Appl. 24 (1968), 677-685.

3. R. Askey and G. Gasper, Jacobi polynomial expansions of Jacobi polynomials with nonnegative coefficients, Proc. Camb. Phil. Soc. 70 (1971), 243-255.

4. H. Bateman, The k-function, a particular case of the confluent hypergeometric function, Trans. Amer. Math. Soc. 33(1931), 817-831.

5. Th. Becker and V. Weispfenning, it Gröbner bases. A computational approach to commutative algebra, Springer, New York, 1991.

6. C. Canuto, M.Y. Hussaini, A. Quarteroni and T.A. Zang, Spectral methods in fiuid dynamics, Springer Series in Computational Physics, New York-Berlin, 1988.

7. F. Chyzak, Holonomic systems and automatic proofs of identities, Rapport de Recherche 2371, INRIA Research Report, Rocquericourt, 1994, available via anonymous ftp on ftp.inria.fr.

8. P. Deuflhard and M. Wulkow, Computational treatment of polyreaction kinetics by orthogonal polynomials of a discrete variable, IMPACT 1, 1989, 269-301.

9. A. Erdélyi, Higher transcendental functions, 2, McGraw Hill Book Co., Inc., New York, 1953.

10. J. Fleischer, O.V. Tarasov, Calculation of Feynman diagrams from their small momentum expansion, Z. Phys. C64, 1994, 413.

11. J. Fröhlich, Parameter derivatives of the Jacobi polynomials and the Gaussian hypergeometric function, Integral Transforms and Special Functions 2, 1994, 252-266.

12. A.C. Hearn, Reduce User's Manual, Version 3.6. RAND Co., Santa Monica, CA, 1995.

13. A. Kandri-Rody and V. Weispfenning, Non-commutative Gröbner bases in algebras of solvable type, J. Symbolic Computation 9, 1990, 1-26.

14. W. Koepf, Algorithmic work with orthogonal polynomials and special functions, KonradZuse-Zentrum Berlin (ZIB), Preprint SC 94-5, 1994.

15. W. Koepf, Algorithms for m-fold hypergeometric summation, J. Symbolic Computation, to appear.

16. W. Koepf, REDUCE package for indefinite and definite summation, SIGSAM Bulletin 29 , $1995,14-30$.

17. W. Koepf, A MATHEMATiCA package on orthogonal polynomials and special functions, in preparation.

18. W. Koepf, Algorithmic Summation and Special Function Identities with Maple, to appear.

19. W. Koepf and D. Schmersau, Bounded nonvanishing functions and Bateman functions, Complex Variables 25, 1994, 237-259.

20. W. Koepf and D. Schmersau, Spaces of functions satisfying simple differentiol equations, Konrad-Zuse-Zentrum Berlin (ZIB), Technical Report TR 94-2, 1994.

21. T.H. Koornwinder, On Zeilberger's algorithm and its q-analogue: a rigorous description, J. of Comput. and Appl. Math. 48, 1993, 91-111.

22. P. Lesky, Unendliche orthogonale Matrizen und Laguerresche Matrizen, Monatsh. Math.63, $1958,59-83$.

23. P. Lesky, Die Übersetzung der klassischen orthogonalen Polynome in die Differenzenrechnung, Monatsh. Math. 65, 1961, 1-26.

24. H. Melenk and J. Apel, REDuCE package NCPOLY: Computation in non-commutative polynomial ideals, Konrad-Zuse-Zentrum Berlin (ZIB), 1994.

25. A.F. Nikiforov, S.K. Suslov and V.B. Uvarov, Classical orthogonal polynomials of a discrete variable, Springer-Verlag, Berlin-Heidelberg-New York, 1991.

26. P. Paule and M. Schorn, $A$ MATHEMATICA VERsion OF ZEILBERGER'S ALGORITHM FOR PROVING BINOMIAL COEFFICIENT IDENTITIES, J. Symbolic Computation, to appear 1995.

27. E.D. Rainville, Special functions, The MacMillan Co., New York, 1960. 
28. B. Salvy and P. Zimmermann, GFUN: A package for the manipulation of generating and holonomic functions in one variable, ACM Transactions on Mathematical Software 20, 1994, 163-177.

29. R.P. Stanley, Differentiably finite power series, Europ. J. Combinatorics 1, 1980, 175-188.

30. G. Szegö, Orthogonal Polynomials, Amer. Math. Soc. Coll. Publ. 23, New York City, 1939.

31. N. Takayama, Gröbner basis and the problem of contiguous relations, Japan J. Appl. Math. 6, 1989, 147-160.

32. N. Takayama, An algorithm of constructing the integral of a module-an infinite dimensional analog of Gröbnet basis, Proc. of ISSAC 90, ACM Press, New York, 1990, 206-211.

33. N. Takayama, Gröbner basis, integration and transcendental functions, Proc. of ISSAC 90 , ACM Press, New York, 1990, 152-156.

34. F.G. Tricomi, Vorlesungen über Orthogonalreihen, Grundlehren der Mathematischen Wissenschaften, 76, Springer-Verlag, Berlin-Göttingen-Heidelberg, 1955.

35. M. Wulkow, Numerical treatment of countable systems of ordinary differential equations, Konrad-Zuse-Zentrum Berlin (ZIB), Technical Report TR 90-8, 1990.

36. D. Zeilberger, A holonomic systems approach to special functions identities, J. Comput. Appl. Math. 32, 1990, 321-368.

37. D. Zeilberger, A fast algorithm for proving terminating hypergeometric identities, Discrete Math. 80, 1990, 207-211.

38. D. Zeilberger, The method of creative telescoping, J. Symbolic Computation 11, 1991, 195204.

39. D. Zeilberger, Three recitations on holonomic systems and hypergeometric series, Proc. of the 24th Séminaire Lotharingen. Ed. by D. Foata, Publ. I. R. M. A. Strasbourg, 5-37. 